\section{External trade and investment}

\section{External trade}

\section{(Table 19.1 and 19.3 to 19.6 )}

The statistics in this section are on a Balance of Payments (BoP) basis, compiled from information provided to HM Revenue and Customs (HMRC) by importers and exporters on an Overseas Trade Statistics (OTS) basis, which values exports 'f.o.b.' (free on board) and imports 'c.i.f.' (including insurance and freight). In addition to deducting these freight costs and insurance premiums from the OTS figures, coverage adjustments are made to convert the OTS data to a BoP basis. Adjustments are also made to the level of all exports and European Union (EU) imports to take account of estimated under-recording. The adjustments are set out and described in the annual United Kingdom Balance of Payments Pink Book (Office for National Statistics (ONS)). These adjustments are made to conform to the definitions in the 5th edition of the IMF Balance of Payments Manual.

Aggregate estimates of trade in goods, seasonally adjusted and on a BoP basis, are published monthly in the ONS First Release UK Trade. More detailed figures are available from time series data on the Office for National Statistics website (www.ons.gov.uk) and are also published in the Monthly Review of External Trade Statistics (Business Monitor MM24). Detailed figures for EU and non-EU trade on an OTS basis are published in Overseas trade statistics: United Kingdom trade with the European Community and the world (HMRC).

A fuller description of how trade statistics are compiled can be found in Statistics on Trade in Goods (Government Statistical Service Methodological Series) available at: www.statistics.gov. uk/STATBASE/Product.asp?vlnk=14943.

\section{Overseas Trade Statistics}

HM Revenue and Customs provide accurate and up to date information via the website: www.uktradeinfo.com They also produce publications 'Overseas Trade Statistics'.

\section{Import penetration and export sales ratios}

(Table 19.2)

The ratios were first introduced in the August 1977 edition of Economic Trends in an article 'The Home and Export
Performance of United Kingdom Industries'. The article described the conceptual and methodological problems involved in measuring such variables as import penetration.

The industries are grouped according to the 1992 Standard Industrial Classification. The four different ratios are defined as follows:

Ratio 1: percentage ratio of imports to home demand

Ratio 2: percentage ratio of imports to (home demand plus exports)

Ratio 3: percentage ratio of exports to total manufacturers' sales

Ratio 4: percentage ratio of exports to (total manufacturers' sales plus imports)

Home demand is defined as total manufacturers' sales plus imports minus exports. This is only an approximate estimate as different sources are used for the total manufacturers' sales and the import and export data. Total manufacturers' sales are determined by the Products of the European Community inquiry, and import and export data are provided by HM Revenue and Customs.

Ratio 1 is commonly used to describe the import penetration of the home market. Allowance is made for the extent of a domestic industry's involvement in export markets by using Ratio 2; this reduces as exports increase.

Similarly, Ratio 3 is the measure normally used to relate exports to total sales by UK producers, and Ratio 4 makes an allowance for the extent that imports of the same product are coming into the UK.

\section{International trade in services}

\section{(Tables 19.7 and 19.8)}

These data relate to overseas trade in services and cover both production and non-production industries (excluding the public sector). In terms of types of services traded, this equates to trade in royalties, various forms of consultancy, computing and telecommunications services, advertising and market research and other business services. A separate inquiry covers the film and television industries. The surveys cover receipts from the provision of services to residents of other countries (exports) and payments to residents of other countries for services rendered (imports). 


\section{Sources of data}

The International Trade in Services (ITIS) surveys (which consist of a quarterly component addressed to the largest businesses and an annual component for the remainder) are based on a sample of companies derived from the Interdepartmental Business Register. The companies are asked to show the amounts for their imports and exports against the geographical area to which they were paid or from which they were received - irrespective of where they were first earned.

The purpose of the ITIS survey is to record international transactions which impact on the UK's BoP; hence companies are asked to exclude from their earnings trade expenses such as the cost of services purchased abroad. Exports and imports of services are excluded where they are included within an invoice for the import or export of goods; in this case they will already be counted in the estimate for Trade in Goods. However, earnings from third country trade - that is, from arranging the sale of goods between two countries other than the UK and where the goods never physically enter the UK (known as merchanting) - are included. Earnings from commodity trading are also included. Together, these two comprise 'Trade Related Services'.

'Royalties' are the largest part of the total trade in services collected in the ITIS survey. These cover transactions for items such as printed matter, sound recordings, performing rights, patents, licences, trademarks, designs, copyrights, manufacturing rights, the use of technical 'know-how' and technical assistance.

\section{Balance of payments}

\section{(Tables 19.9 to 19.12 )}

Tables 19.9 to 19.12 are derived from United Kingdom Balance of Payments: The Pink Book 2008 edition. The following general notes to the tables provide brief definitions and explanations of the figures and terms used. Further notes are included in the Pink Book.

\section{Summary of Balance of Payments}

The BoP consists of the current account, the capital account, the financial account and the International Investment Position (IIP). The current account consists of trade in goods and services, income and current transfers. Income consists of investment income and compensation of employees. The capital account mainly consists of capital transfers and the financial account covers financial transactions. The IIP covers balance sheet levels of UK external assets and liabilities. Every credit entry in the balance of payments accounts should, in theory, be matched by a corresponding debit entry so that total current, capital and financial account credits should be equal to, and therefore offset by, total debits. In practice there is a discrepancy termed net errors and omissions.

\section{The current account}

\section{Trade in goods}

The goods account covers exports and imports of goods. Imports of motor cars from Japan, for example, are recorded as debits in the trade in goods account, whereas exports of vehicles manufactured in the UK are recorded as credits. Trade in goods forms a component of the expenditure measure of Gross Domestic Product (GDP).

\section{Trade in services}

The services account covers exports and imports of services (for example, civil aviation). Passenger tickets for travel on UK aircraft sold abroad, for example, are recorded as credits in the services account, whereas the purchases of airline tickets from foreign airlines by UK passengers are recorded as debits. Trade in services, along with trade in goods, forms a component of the expenditure measure of GDP.

\section{Income}

The income account consists of compensation of employees and investment income and is dominated by the latter. Compensation of employees covers employment income from cross-border and seasonal workers which is less significant in the UK than in other countries. Investment income covers earnings (for instance, profits, dividends and interest payments and receipts) arising from cross-border investment in financial assets and liabilities. For example, earnings on foreign bonds and shares held by financial institutions based in the UK are recorded as credits in the investment income account, whereas earnings on UK company securities held abroad are recorded as investment income debits. Investment income forms a component of Gross National Income (GNI) but not GDP.

\section{Current transfers}

Current transfers are composed of central government transfers (for instance, taxes and payments to, and receipts from, the EU) and other transfers (for instance, gifts in cash or kind received by private individuals from abroad or receipts from the EU, where the UK government acts as an agent for the ultimate beneficiary of the transfer). Current transfers do not form a component either of GDP or of GNI. For example, payments to the UK farming industry under the EU Agricultural Guarantee Fund are recorded as credits in the current transfers account, while payments of EU agricultural 
levies by the UK farming industry are recorded as debits in the current transfers account.

\section{Capital account}

Capital account transactions involve transfers of ownership of fixed assets, transfers of funds associated with acquisition or disposal of fixed assets, and cancellation of liabilities by creditors without any counterparts being received in return. The main components are migrants transfers, EU transfers relating to fixed capital formation (regional development fund and agricultural guidance fund) and debt forgiveness. Funds brought into the UK by new immigrants would, for example, be recorded as credits in the capital account, while funds sent abroad by UK residents emigrating to other countries would be recorded as debits in the capital account. The size of capital account transactions are quite minor compared with the current and financial accounts.

\section{Financial account}

While investment income covers earnings arising from crossborder investments in financial assets and liabilities, the financial account of the balance of payments covers the flows of such investments. Earnings on foreign bonds and shares held by financial institutions based in the UK are, for example, recorded as credits in the investment income account, but the acquisition of such foreign securities by UK-based financial institutions are recorded as net debits in the financial account or portfolio investment abroad. Similarly, the acquisitions of UK company securities held by foreign residents are recorded in the financial account as net credits or portfolio investment in the UK.

\section{International Investment Position}

While the financial account covers the flows of foreign investments and financial assets and liabilities, the IIP records the levels of external assets and liabilities. While the acquisition of foreign securities by UK-based financial institutions are recorded in the financial account, as net debits, the total holdings of foreign securities by UK-based financial institutions are recorded as levels of UK external assets. Similarly, the holdings of UK company securities held by foreign residents are recorded as levels of UK liabilities.

\section{Foreign direct investment}

\section{(Tables 19.13 to 19.18 )}

Direct investment refers to investment that adds to, deducts from or acquires a lasting interest in an enterprise operating in an economy other than that of the investor, the investor's purpose being to have an effective voice in the management of the enterprise. (For the purposes of the statistical inquiry, an effective voice is taken as equivalent to a holding of 10 per cent or more in the foreign enterprise.) Other investments in which the investor does not have an effective voice in the management of the enterprise are mainly portfolio investments and these are not covered here. Cross-border investment by public corporations or in property (which is regarded as direct investment in the national accounts) are not covered here, but are shown in the BoP. Similarly, foreign direct investment earnings data are shown net of tax in Tables 19.15 and 19.18 but are gross of tax in the BoP.

Direct investment is a financial concept and is not the same as capital expenditure on fixed assets. It covers only the money invested in a related concern by the parent company, and the concern will then decide how to use the money. A related concern may also raise money locally without reference to the parent company.

The investment figures are published on a net basis; that is, they consist of investments net of disinvestments by a company into its foreign subsidiaries, associate companies and branches.

\section{Definitional changes from 1997}

The new European System of Accounts (ESA(95)) definitions were introduced from the 1997 estimates. The changes were as follows:

i) Previously for the measurement of direct investment, an effective voice in the management of an enterprise was taken as the equivalent of a 20 per cent shareholding. This is now 10 per cent.

ii) The Channel Islands (Jersey, Guernsey, etc.) and the Isle of Man have been excluded from the definition of the economic territory of the UK. Prior to 1987, these islands were considered to be part of the UK.

iii) Interest received or paid was replaced by interest accrued in the figures on earnings from direct investment. There is deemed to be little or no impact arising from this definitional change on the estimates.

New register sources available from 1998 have led to revisions for the figures from that year onwards. These sources gave an improved estimate of the population satisfying the criteria for foreign direct investment.

The definitional changes have been introduced from 1997 and the register changes from 1998. The data prior to these 
years have not been reworked in Tables 19.13 to 19.18 . For clarity, the Offshore Islands are identified separately on the tables. The breaks in the series for the other definitional changes are not quantified but are relatively small. More detailed information on the effect of these changes appears in the business monitor MA4 - Foreign Direct Investment 2002, which was published in February 2003 and is available from the Office for National Statistics website.

\section{Sources of data}

The figures in Tables 19.13 to 19.18 are based on annual inquiries into foreign direct investment for 2007. These were sample surveys which involved sending around 1,250 forms to UK businesses investing abroad, and 2,250 forms to UK businesses in which foreign parents and associates had invested. The tables also contain some revisions to 2006 as a result of new information coming to light in the course of the latest surveys. Further details from the latest annual surveys, including analyses by industry and by components of direct investment, are available in business monitor MA4. Initial figures were published on the Office for National Statistics website in a First Release Foreign Direct Investment 2007 in December 2008. Data for 2008 will be published in a First Release in December 2009, followed by the full business monitor MA4 in February 2009.

\section{Country allocation}

The analysis of inward investment is based on the country of ownership of the immediate parent company. Thus, inward investment in a UK company may be attributed to the country of the intervening overseas subsidiary, rather than the country of the ultimate parent. Similarly, the country analysis of outward investment is based on the country of ownership of the immediate subsidiary. As an example, to the extent that overseas investment in the UK is channelled through holding companies in the Netherlands, the underlying flow of investment from this country is overstated and the inflow from originating countries is understated.

\section{Further information}

More detailed statistics on foreign direct investment are available on request from Richard Tonkin, Office for National Statistics, International Transactions Branch, Room 2.354, Government Buildings, Cardiff Road, Newport, South Wales, United Kingdom, NP10 8XG. Telephone: +44 (0)1633 456082, fax: +44 (0)1633 812855, email Richard.tonkin@ons.gov.uk. 
External trade and investment

\begin{tabular}{|c|c|c|c|c|c|c|c|c|c|c|c|c|}
\hline & & 1998 & 1999 & 2000 & 2001 & 2002 & 2003 & 2004 & 2005 & 2006 & 2007 & 2008 \\
\hline \multicolumn{13}{|l|}{ Value ( $£$ million) } \\
\hline Exports of goods & BOKG & 164056 & 166166 & 187936 & 189093 & 186524 & 188320 & 190874 & 211608 & 243635 & 220858 & 251088 \\
\hline Imports of goods & $\mathrm{BOKH}$ & 185869 & 195217 & 220912 & 230305 & 234229 & 236927 & 251774 & 280197 & 319947 & 310612 & 343964 \\
\hline Balance on trade in goods & BOKI & -21813 & -29051 & -32976 & -41212 & -47705 & -48607 & -60900 & -68589 & -76312 & -89754 & -92876 \\
\hline \multicolumn{13}{|l|}{ Price index numbers } \\
\hline Exports of goods & BQKR & 100.9 & 98.8 & 99.9 & 98.3 & 98.2 & 100.0 & 100.0 & 104.6 & 107.8 & 109.2 & 125.9 \\
\hline Imports of goods & BQKS & 102.4 & 100.8 & 104.2 & 103.3 & 100.7 & 100.0 & 99.4 & 103.7 & 107.5 & 109.0 & 123.1 \\
\hline Terms of trade ${ }^{2}$ & $\mathrm{BQKT}$ & 98.5 & 98.0 & 95.9 & 95.2 & 97.5 & 100.0 & 100.6 & 100.9 & 100.3 & 100.2 & 102.3 \\
\hline \multicolumn{13}{|l|}{ Volume index numbers } \\
\hline Exports of goods & BQKU & 85.8 & 88.6 & 99.3 & 101.5 & 100.3 & 100.0 & 101.5 & 111.0 & 125.2 & 110.4 & 110.6 \\
\hline Imports of goods & $\mathrm{BQKV}$ & 76.4 & 81.5 & 89.1 & 93.8 & 98.2 & 100.0 & 106.9 & 114.6 & 127.5 & 122.3 & 119.8 \\
\hline
\end{tabular}

1 See chapter text. Statistics of trade in goods on a balance of payments basis are obtained by making certain adjustments in respect of valuation and Source: Office for National Statistics: 02070142018 coverage to the statistics recorded in the Overseas Trade Statistics. These

adjustments are described in detail in The Pink Book 2008.

2 Export price index as a percentage of the import price index. 
External trade and investment industry ${ }^{1,2}$

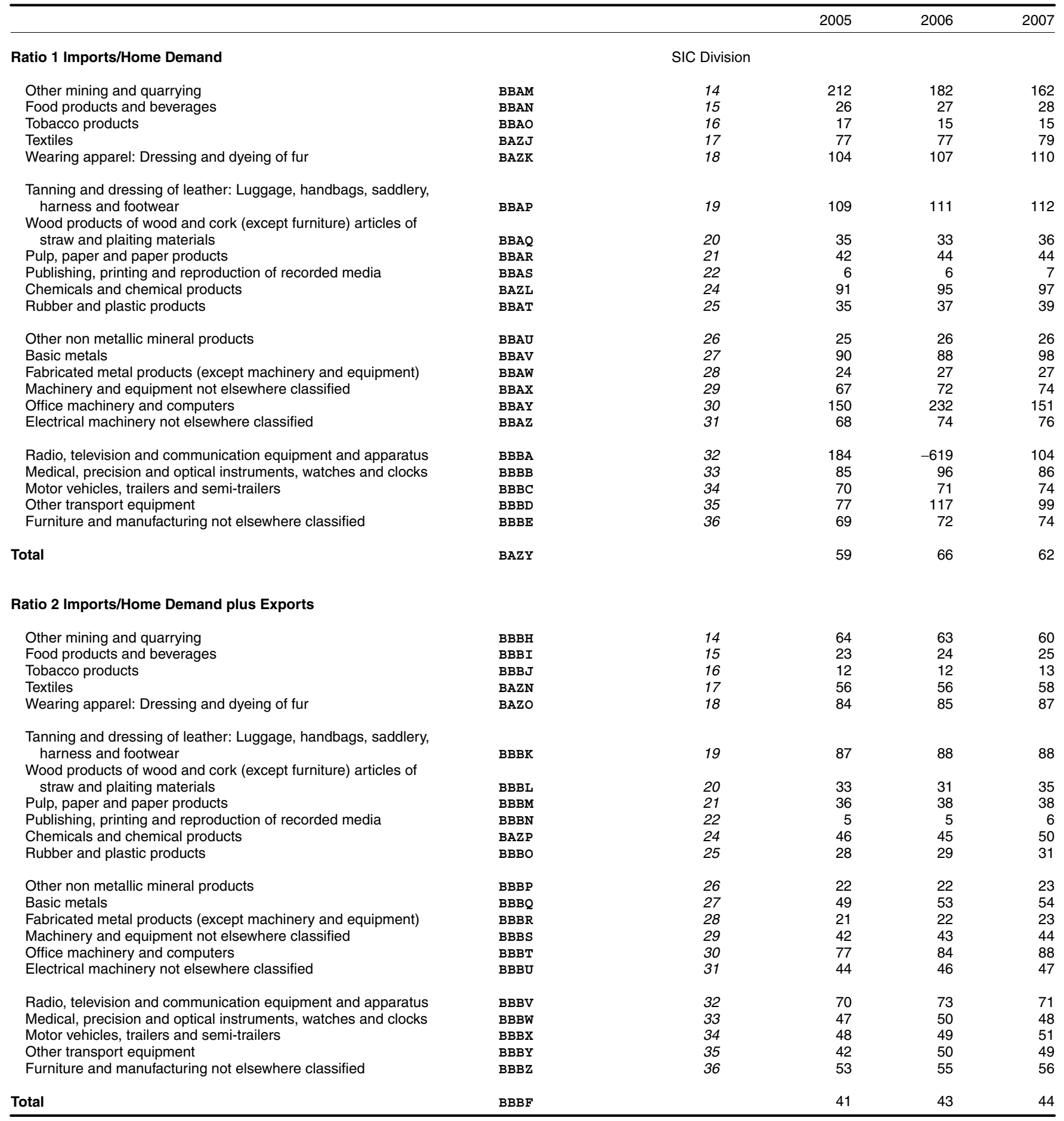




Ratio 3 Exports/Sales
Other mining and quarrying
Food products and beverages
Tobacco products
Textiles
Wearing apparel: Dressing and dyeing of fur
Tanning and dressing of leather: Luggage, handbags, saddlery,
harness and footwear
Wood products of wood and cork (except furniture) articles of
straw and plaiting materials
Pulp, paper and paper products
Publishing, printing and reproduction of recorded media
Chemicals and chemical products
Rubber and plastic products
Other non metallic mineral products
Basic metals
Fabricated metal products (except machinery and equipment)
Machinery and equipment not elsewhere classified
Office machinery and computers
Electrical machinery not elsewhere classified
Radio, television and communication equipment and apparatus
Medical, precision and optical instruments, watches and clocks
Motor vehicles, trailers and semi-trailers
Other transport equipment
Furniture and manufacturing not elsewhere classified
2006

SIC Division

$\mathrm{BBCM}$

$B B C N$

BBCO

BAZR

BAZS

BBCP

BBCQ

BBCR

BBCS

BAZT

BBCT

$\mathrm{BBCU}$

$\mathrm{BBCV}$

$\mathrm{BBCW}$

$B B C X$

BBCY

BBDK

BBDL

BBDM

BBDN

BBDO

BBDP

BBCK

14
15
16
17
18

18

19

20

22

24
25

25

26

27

29

30

31

32

33

34

35
36

\section{Ratio 4 Exports/Sales plus Imports}

Other mining and quarrying

Food products and beverages

Tobacco products

Textiles

Wearing apparel: Dressing and dyeing of fur

Tanning and dressing of leather: Luggage, handbags, saddlery, harness and footwear

BBDV

Wood products of wood and cork (except furniture) articles of

straw and plaiting materials

Pulp, paper and paper products

Publishing, printing and reproduction of recorded media

Chemicals and chemical products

BBDW

BBDX

BBDY

BAZX

Rubber and plastic products

Other non-metallic mineral products

Basic metals

Fabricated metal products (except machinery and equipment)

Machinery and equipment not elsewhere classified

Office machinery and computers

Electrical machinery not elsewhere classified

Radio, television and communication equipment and apparatus

Medical, precision and optical instruments, watches and clocks

Motor vehicles, trailers and semi-trailers

Other transport equipment

Furniture and manufacturing not elsewhere classified

BBDZ

BBEA

BBEB

BBEC

BBED

BBEE

BBEF

$\begin{array}{rrr}195 & 178 & 158 \\ 15 & 15 & 16 \\ 35 & 25 & 18 \\ 62 & 62 & 63 \\ 121 & 136 & 160\end{array}$

63

168

180

BBDQ

1 See chapter text.

2 Division 13 (Mining of metal ores) has not been published since 1995. Division 23 (Coke, refined petroleum products and nuclear fuel) and SIC 24610 (Manufacture of explosives) are excluded from the analysis. SIC 27100 (Basic iron and steel and ferro-alloys) is not incorporated in PRODCOM and therefore also does not form part of the analysis. 


\begin{tabular}{|c|c|c|c|c|c|c|c|c|c|c|c|}
\hline & & 1999 & 2000 & 2001 & 2002 & 2003 & 2004 & 2005 & 2006 & 2007 & 2008 \\
\hline 0. Food and live animals & BOGG & 5925 & 5827 & 5491 & 5693 & 6478 & 6461 & 6552 & 6770 & 7374 & 8726 \\
\hline \multicolumn{12}{|l|}{ of which: } \\
\hline 01. Meat and meat preparations & BOGS & 657 & 642 & 428 & 516 & 606 & 667 & 729 & 754 & 839 & 1173 \\
\hline 02. Dairy products and eggs & BQMS & 689 & 660 & 614 & 625 & 760 & 780 & 718 & 712 & 807 & 900 \\
\hline $04 \& 08$. Cereals and animal feeding stuffs & BQMT & 1568 & 1604 & 1383 & 1444 & 1681 & 1553 & 1554 & 1587 & 1791 & 2286 \\
\hline 05. Vegetables and fruit & BQMU & 437 & 403 & 401 & 433 & 475 & 507 & 515 & 586 & 606 & 696 \\
\hline 1. Beverages and tobacco & BQMZ & 4022 & 4081 & 4139 & 4300 & 4401 & 4116 & 4095 & 4175 & 4395 & 5012 \\
\hline 11. Beverages & BQNB & 3004 & 3065 & 3218 & 3320 & 3478 & 3354 & 3481 & 3715 & 4093 & 4563 \\
\hline 12. Tobacco & BQOW & 1018 & 1016 & 921 & 980 & 923 & 762 & 614 & 460 & 302 & 449 \\
\hline 2. Crude materials & BQOX & 2087 & 2447 & 2422 & 2645 & 3069 & 3565 & 3746 & 4621 & 5196 & 6258 \\
\hline \multicolumn{12}{|l|}{ of which: } \\
\hline 24. Wood, lumber and cork & BQOY & 66 & 72 & 70 & 81 & 106 & 117 & 131 & 146 & 144 & 127 \\
\hline 25. Pulp and waste paper & $\mathrm{BQOZ}$ & 54 & 78 & 81 & 106 & 180 & 244 & 283 & 338 & 417 & 481 \\
\hline 28. Metal ores & $B Q P B$ & 518 & 759 & 810 & 928 & 1193 & 1604 & 1713 & 2418 & 2898 & 3665 \\
\hline 3. Fuels & BOPN & 9929 & 17057 & 16386 & 16000 & 16558 & 17885 & 21496 & 25301 & 24700 & 34846 \\
\hline 33. Petroleum and petroleum products & ELBL & 9123 & 15584 & 14815 & 14321 & 14608 & 16200 & 19794 & 23173 & 22756 & 31666 \\
\hline $32,34 \& 35$. Coal, gas and electricity & BOQI & 806 & 1473 & 1571 & 1679 & 1950 & 1685 & 1702 & 2128 & 1944 & 3180 \\
\hline 4. Animal and vegetable oils and fats & BQPI & 197 & 156 & 149 & 210 & 266 & 205 & 235 & 271 & 327 & 360 \\
\hline 5. Chemicals & ENDG & 23071 & 24992 & 27514 & 28386 & 31373 & 32009 & 33388 & 37179 & 38891 & 43877 \\
\hline \multicolumn{12}{|l|}{ of which: } \\
\hline 51. Organic chemicals & BQPJ & 5494 & 5718 & 6090 & 5698 & 6070 & 6040 & 6702 & 8009 & 7601 & 8391 \\
\hline 52. Inorganic chemicals & $\mathrm{BQPK}$ & 1137 & 1491 & 1636 & 1367 & 1460 & 1543 & 1555 & 2143 & 2830 & 2823 \\
\hline 53. Colouring materials & CSCE & 1534 & 1555 & 1521 & 1583 & 1627 & 1630 & 1635 & 1602 & 1672 & 1827 \\
\hline 54. Medicinal products & BQPL & 6279 & 7217 & 9067 & 10103 & 11897 & 12325 & 12320 & 13786 & 14507 & 17310 \\
\hline 55. Toilet preparations & CSCF & 2462 & 2597 & 2714 & 2823 & 3122 & 3105 & 3219 & 3443 & 3689 & 4051 \\
\hline $57 \&$ 58. Plastics & BQQA & 3144 & 3366 & 3416 & 3526 & 3703 & 3847 & 4298 & 4445 & 4612 & 4926 \\
\hline 6. Manufactures classified chiefly by material & BQQB & 20302 & 22673 & 22781 & 21837 & 23119 & 24458 & 26492 & 27664 & 29378 & 32557 \\
\hline 63. Wood and cork manufactures & $B Q Q C$ & 278 & 255 & 261 & 270 & 322 & 291 & 255 & 273 & 272 & 242 \\
\hline 64. Paper and paperboard manufactures & $B Q Q D$ & 2020 & 2096 & 2081 & 2019 & 2097 & 1996 & 2043 & 2014 & 2124 & 2341 \\
\hline 65. Textile manufactures & $B Q Q E$ & 3020 & 3051 & 3022 & 2847 & 2956 & 2847 & 2647 & 2680 & 2589 & 2598 \\
\hline 67. Iron and steel & $B Q Q F$ & 2576 & 2848 & 2879 & 2916 & 3319 & 4245 & 5183 & 5131 & 6016 & 6867 \\
\hline 68. Non-ferrous metals & BQQG & 2130 & 3171 & 3033 & 2552 & 2567 & 3228 & 3862 & 4827 & 5778 & 6964 \\
\hline 69. Metal manufactures & $B Q Q H$ & 3553 & 3595 & 3853 & 3660 & 3766 & 3856 & 4066 & 4520 & 4665 & 5071 \\
\hline 7. Machinery and transport equipment ${ }^{3}$ & $B Q Q I$ & 78875 & 87812 & 87240 & 84395 & 79650 & 78376 & 89379 & 110394 & 82713 & 89234 \\
\hline $71-716,72,73 \&$ \&4. Mechanical machinery & BQQK & 21888 & 22140 & 24244 & 22704 & 24231 & 23808 & 25795 & 28245 & 28969 & 32232 \\
\hline $716,75,76 \& 77$. Electrical machinery & BQQL & 36012 & 42681 & 41997 & 38706 & 30651 & 28624 & 37120 & 55336 & 24215 & 25325 \\
\hline 78. Road vehicles & BQQM & 15077 & 15604 & 13845 & 16316 & 17474 & 18489 & 19439 & 19334 & 21114 & 22551 \\
\hline 79. Other transport equipment & $B Q Q N$ & 5898 & 7387 & 7154 & 6669 & 7294 & 7455 & 7025 & 7479 & 8415 & 9126 \\
\hline 8. Miscellaneous manufactures ${ }^{3}$ & BQQO & 20263 & 21206 & 21948 & 21985 & 22543 & 22917 & 25105 & 25974 & 26695 & 28453 \\
\hline \multicolumn{12}{|l|}{ of which: } \\
\hline 84. Clothing & $\operatorname{CsCN}$ & 2804 & 2722 & 2578 & 2507 & 2708 & 2729 & 2712 & 2877 & 3100 & 3307 \\
\hline 85. Footwear & CSCP & 532 & 514 & 484 & 452 & 426 & 419 & 470 & 522 & 541 & 624 \\
\hline $87 \& 88$. Scientific and photographic & BQQQ & 6732 & 7333 & 7775 & 7212 & 7281 & 7040 & 7245 & 7344 & 7063 & 8061 \\
\hline 9. Other commodities and transactions & BOQL & 1495 & 1685 & 1023 & 1073 & 863 & 882 & 1120 & 1286 & 1189 & 1765 \\
\hline Total United Kingdom exports & BOKG & 166166 & 187936 & 189093 & 186524 & 38320 & 190874 & 211608 & 243635 & 220858 & 251088 \\
\hline
\end{tabular}

1 See chapter text. The numbers on the left hand side of the table refer to the

3, which was introduced in January 1988.

2 Balance of payments consistent basis.

3 Sections 7 and 8 are shown by broad economic category in table G2 of the

Monthly Review of External Trade Statistics. 


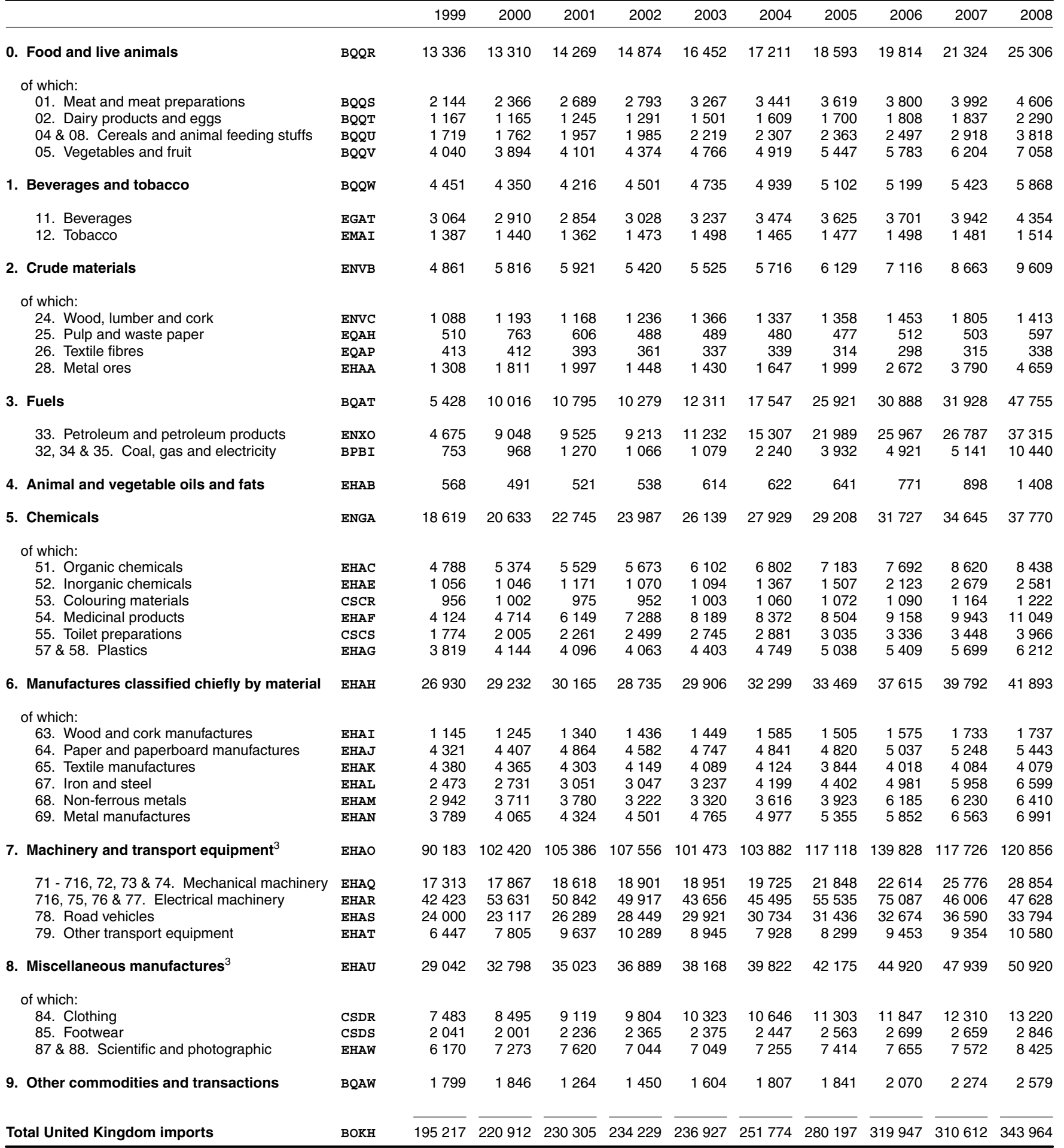

1 See chapter text. The numbers on the left hand side of the table refer to the

3 , which was introduced in January 1988.

2 Balance of payments consistent basis.

3 Sections 7 and 8 are shown by broad economic category in table G2 of the

Monthly Review of External Trade Statistics. 
$£$ million

\begin{tabular}{|c|c|c|c|c|c|c|c|c|c|c|c|}
\hline & & 1999 & 2000 & 2001 & 2002 & 2003 & 2004 & 2005 & 2006 & 2007 & 2008 \\
\hline European Union: ${ }^{3}$ & LGCK & 101537 & 112459 & 114406 & 114737 & 111286 & 111650 & 121486 & 152357 & 127813 & 141136 \\
\hline EMU members: & QAKW & 92359 & 102138 & 104202 & 103908 & 100631 & 100595 & 109506 & 136061 & 114537 & 126102 \\
\hline Austria & CHMY & 1168 & 1146 & 1224 & 1265 & 1264 & 1095 & 1332 & 1699 & 1376 & 1473 \\
\hline Belgium \& Luxembourg & CHNQ & 9241 & 10322 & 9893 & 10552 & 11374 & 10510 & 11394 & 15082 & 12122 & 13266 \\
\hline Finland & CHMZ & 1354 & 1471 & 1611 & 1442 & 1493 & 1363 & 1514 & 1872 & 1958 & 1908 \\
\hline France & ENYL & 16907 & 18577 & 19249 & 18757 & 18885 & 18562 & 19931 & 28693 & 18103 & 18067 \\
\hline Germany & ENYO & 20464 & 22789 & 23655 & 22064 & 20805 & 21668 & 23025 & 27602 & 24699 & 28062 \\
\hline Greece & CHNT & 1181 & 1267 & 1156 & 1234 & 1286 & 1408 & 1367 & 1469 & 1350 & 1649 \\
\hline Irish Republic & CHNS & 10783 & 12372 & 13835 & 15422 & 12224 & 14134 & 16294 & 17480 & 17801 & 19104 \\
\hline Italy & CHNO & 7831 & 8429 & 8404 & 8506 & 8603 & 8400 & 8790 & 9494 & 9189 & 9351 \\
\hline Netherlands & CHNP & 13632 & 15167 & 14599 & 14011 & 13597 & 12029 & 12716 & 16522 & 15115 & 19697 \\
\hline Portugal & CHNU & 1712 & 1660 & 1579 & 1518 & 1453 & 1580 & 1698 & 2374 & 1481 & 1640 \\
\hline Spain & CHNV & 7526 & 8302 & 8363 & 8490 & 8943 & 9100 & 10677 & 12295 & 9979 & 10227 \\
\hline Non-EMU members: ${ }^{3}$ & BQIA & 9178 & 10321 & 10204 & 10829 & 10655 & 11055 & 11980 & 16296 & 13276 & 15034 \\
\hline Bulgaria & WYUF & 76 & 85 & 122 & 134 & 154 & 155 & 220 & 237 & 202 & 252 \\
\hline Czech Rep & FKML & 733 & 927 & 1075 & 1031 & 1003 & 978 & 1080 & 1526 & 1401 & 1547 \\
\hline Denmark & CHNR & 2054 & 2315 & 2267 & 2729 & 2180 & 2042 & 2314 & 3715 & 2182 & 2599 \\
\hline Hungary & QALC & 486 & 613 & 612 & 750 & 856 & 934 & 834 & 855 & 863 & 1009 \\
\hline Poland & ERDR & 1169 & 1299 & 1297 & 1318 & 1462 & 1417 & 1653 & 2705 & 2372 & 3010 \\
\hline Romania & WMDB & 242 & 381 & 341 & 432 & 509 & 609 & 647 & 637 & 668 & 755 \\
\hline Sweden & CHNA & 4035 & 4211 & 3951 & 3873 & 3823 & 4356 & 4588 & 5246 & 4904 & 5194 \\
\hline Other Western Europe: & HCJD & 6244 & 7223 & 6786 & 6334 & 6629 & 7031 & 9730 & 9221 & 9232 & 10846 \\
\hline \multicolumn{12}{|l|}{ of which: } \\
\hline Iceland & EPLW & 159 & 193 & 150 & 131 & 141 & 167 & 179 & 188 & 198 & 189 \\
\hline Norway & EPLX & 1999 & 2018 & 1813 & 1696 & 1886 & 1939 & 2211 & 2125 & 2697 & 2875 \\
\hline Switzerland & EPLV & 2768 & 3061 & 3496 & 3080 & 2786 & 2842 & 4985 & 4189 & 3808 & 4701 \\
\hline Turkey & EOBA & 1198 & 1800 & 1150 & 1287 & 1638 & 1903 & 2160 & 2426 & 2283 & 2580 \\
\hline North America: & HBZQ & 27582 & 33714 & 33408 & 32261 & 32924 & 32764 & 35010 & 36929 & 36367 & 39416 \\
\hline \multicolumn{12}{|l|}{ of which: } \\
\hline Canada & ЕОВС & 2532 & 3487 & 3203 & 3107 & 3239 & 3340 & 3277 & 3894 & 3291 & 3282 \\
\hline Other OECD countries: & HCII & 6728 & 8028 & 7542 & 7469 & 7824 & 8226 & 8577 & 8716 & 8778 & 10011 \\
\hline \multicolumn{12}{|l|}{ of which: } \\
\hline Australia & EPMA & 2155 & 2699 & 2298 & 2114 & 2289 & 2455 & 2580 & 2488 & 2630 & 3122 \\
\hline Japan & EOBD & 3300 & 3672 & 3673 & 3583 & 3710 & 3863 & 3900 & 4109 & 3866 & 3933 \\
\hline New Zealand & EPMB & 324 & 305 & 309 & 311 & 348 & 418 & 415 & 373 & 364 & 388 \\
\hline South Korea & ERDM & 949 & 1350 & 1262 & 1461 & 1468 & 1481 & 1677 & 1746 & 1914 & 2568 \\
\hline Oil exporting countries: & HDII & 5524 & 6031 & 6474 & 6229 & 7615 & 7996 & 10850 & 9060 & 9716 & 11693 \\
\hline \multicolumn{12}{|l|}{ of which: } \\
\hline Brunei & QALF & 124 & 96 & 59 & 61 & 127 & 67 & 43 & 79 & 870 & 66 \\
\hline Dubai & QALI & 790 & 966 & 1012 & 940 & 1383 & 2019 & 4656 & 2829 & 1927 & 2718 \\
\hline Indonesia & FKMR & 385 & 404 & 313 & 324 & 452 & 397 & 366 & 311 & 289 & 390 \\
\hline Kuwait & QATB & 293 & 338 & 359 & 308 & 373 & 354 & 426 & 438 & 450 & 547 \\
\hline Nigeria & QATE & 447 & 524 & 686 & 711 & 738 & 773 & 799 & 821 & 1043 & 1530 \\
\hline Saudi Arabia & ERDI & 1481 & 1557 & 1525 & 1388 & 1819 & 1611 & 1559 & 1644 & 1857 & 2211 \\
\hline Rest of the World & HCHW & 18551 & 20481 & 20477 & 19494 & 22042 & 23207 & 25955 & 27352 & 28952 & 37986 \\
\hline \multicolumn{12}{|l|}{ of which: } \\
\hline Brazil & FKMO & 739 & 775 & 808 & 880 & 825 & 789 & 836 & 918 & 1108 & 1704 \\
\hline China & ERDN & 1211 & 1468 & 1709 & 1493 & 1924 & 2366 & 2811 & 3264 & 3860 & 5117 \\
\hline Egypt & QALL & 539 & 498 & 452 & 463 & 458 & 667 & 543 & 577 & 686 & 951 \\
\hline Hong Kong & ERDG & 2312 & 2673 & 2683 & 2411 & 2481 & 2630 & 3087 & 2864 & 2726 & 3702 \\
\hline India & ERDJ & 1450 & 2058 & 1772 & 1755 & 2284 & 2234 & 2798 & 2693 & 2968 & 4164 \\
\hline Israel & ERDL & 1295 & 1516 & 1357 & 1428 & 1359 & 1386 & 1352 & 1308 & 1257 & 1350 \\
\hline Malaysia & ERDK & 934 & 907 & 1029 & 877 & 1028 & 991 & 1088 & 877 & 975 & 1142 \\
\hline Pakistan & FKMU & 221 & 207 & 229 & 240 & 291 & 343 & 461 & 488 & 423 & 479 \\
\hline Philippines & FKMX & 239 & 273 & 392 & 352 & 377 & 315 & 279 & 242 & 251 & 246 \\
\hline Russia & ERDQ & 532 & 668 & 893 & 981 & 1420 & 1465 & 1869 & 2063 & 2893 & 4305 \\
\hline Singapore & ERDH & 1597 & 1625 & 1592 & 1445 & 1582 & 1708 & 2078 & 2318 & 2467 & 2840 \\
\hline South Africa & EPME & 1281 & 1413 & 1534 & 1597 & 1766 & 1874 & 2073 & 2184 & 2244 & 2675 \\
\hline Taiwan & ERDP & 865 & 1015 & 875 & 848 & 897 & 950 & 939 & 911 & 957 & 896 \\
\hline Thailand & ERDO & 463 & 582 & 594 & 529 & 572 & 637 & 638 & 567 & 613 & 760 \\
\hline
\end{tabular}

1 See chapter text.

2 Balance of payments consistent basis.

Source: Office for National Statistics: 02070142018

3 Includes Bulgaria and Romania after accession on 1 January 2007. 


\begin{tabular}{|c|c|c|c|c|c|c|c|c|c|c|c|}
\hline & & 1999 & 2000 & 2001 & 2002 & 2003 & 2004 & 2005 & 2006 & 2007 & 2008 \\
\hline European Union: ${ }^{3}$ & LGDC & 109622 & 117644 & 126973 & 136931 & 137404 & 142523 & 158163 & 183748 & 169799 & 180322 \\
\hline EMU members & QAKX & 99645 & 106146 & 114724 & 123716 & 123224 & 126804 & 139541 & 157276 & 149719 & 157346 \\
\hline Austria & CHNB & 1453 & 1410 & 1888 & 2396 & 2776 & 2354 & 2461 & 2786 & 2488 & 2327 \\
\hline Belgium \& Luxembourg & CHNY & 10156 & 10927 & 12159 & 13201 & 13205 & 13846 & 15155 & 18183 & 15820 & 17178 \\
\hline Finland & CHNC & 2365 & 2765 & 2965 & 2791 & 2663 & 2336 & 2431 & 3118 & 2619 & 2777 \\
\hline France & ENYP & 18410 & 18644 & 20127 & 20798 & 20389 & 20133 & 21984 & 26376 & 21896 & 23154 \\
\hline Greece & СНОВ & 408 & 459 & 476 & 555 & 613 & 637 & 703 & 789 & 640 & 663 \\
\hline Irish Republic & CHOA & 8705 & 10261 & 12141 & 13176 & 9920 & 10131 & 10411 & 10770 & 11338 & 12286 \\
\hline Italy & CHNW & 9383 & 9514 & 9860 & 10675 & 11481 & 12184 & 12673 & 12775 & 13316 & 13891 \\
\hline Netherlands & CHNX & 13768 & 15380 & 15395 & 16143 & 16692 & 18196 & 20436 & 22275 & 23079 & 25715 \\
\hline Portugal & $\mathrm{CHOC}$ & 1822 & 1735 & 1625 & 1761 & 1966 & 1928 & 2018 & 3054 & 1506 & 1738 \\
\hline Spain & CHOD & 5966 & 6141 & 7360 & 9190 & 9247 & 9120 & 11450 & 12144 & 10489 & 10745 \\
\hline Non-EMU members: ${ }^{3}$ & BQIB & 9977 & 11498 & 12249 & 13215 & 14180 & 15719 & 18622 & 26472 & 20080 & 22976 \\
\hline \multicolumn{12}{|l|}{ of which: } \\
\hline Bulgaria & WYUT & 69 & 85 & 101 & 116 & 124 & 150 & 169 & 208 & 239 & 209 \\
\hline Czech Rep & FKMM & 580 & 802 & 1097 & 1250 & 1412 & 1291 & 1883 & 2987 & 2983 & 3563 \\
\hline Denmark & CHNZ & 2341 & 2630 & 2922 & 3595 & 3399 & 3357 & 4393 & 6439 & 3444 & 3905 \\
\hline Hungary & QALD & 668 & 683 & 710 & 846 & 1120 & 1579 & 1860 & 2348 & 2377 & 2532 \\
\hline Poland & ERED & 676 & 905 & 1166 & 1265 & 1545 & 1835 & 2320 & 3622 & 3695 & 4330 \\
\hline Romania & WMDC & 253 & 336 & 448 & 522 & 679 & 786 & 803 & 861 & 938 & 762 \\
\hline Sweden & CHND & 4648 & 4951 & 4671 & 4330 & 4568 & 5118 & 5463 & 5985 & 5274 & 6805 \\
\hline Other Western Europe: & HBTS & 10554 & 13040 & 12240 & 12523 & 13331 & 15754 & 20072 & 23417 & 24359 & 32496 \\
\hline \multicolumn{12}{|l|}{ of which: } \\
\hline Iceland & EPMW & 282 & 365 & 281 & 289 & 296 & 355 & 346 & 402 & 415 & 458 \\
\hline Norway & EPMX & 3546 & 5563 & 5523 & 5258 & 6423 & 8495 & 12077 & 14453 & 14316 & 21650 \\
\hline Switzerland & EPMV & 5341 & 5485 & 4544 & 4595 & 3759 & 3447 & 3884 & 4372 & 4746 & 5264 \\
\hline Turkey & EOBU & 1204 & 1450 & 1669 & 2164 & 2619 & 3250 & 3510 & 3946 & 4632 & 4885 \\
\hline North America: & HCRB & 28035 & 33460 & 34617 & 29811 & 27480 & 27130 & 27133 & 31228 & 32470 & 32468 \\
\hline \multicolumn{12}{|l|}{ of which: } \\
\hline Canada & EOBW & 3026 & 4009 & 3664 & 3563 & 3664 & 4194 & 4157 & 4954 & 5793 & 5796 \\
\hline Other OECD countries: & HDJQ & 13805 & 15717 & 14154 & 13017 & 12989 & 13644 & 14424 & 13633 & 13870 & 15171 \\
\hline \multicolumn{12}{|l|}{ of which: } \\
\hline Australia & EPNA & 1338 & 1543 & 1776 & 1688 & 1789 & 1868 & 2100 & 2107 & 2245 & 2386 \\
\hline Japan & EOBX & 9118 & 10214 & 9080 & 8079 & 8085 & 8109 & 8669 & 7857 & 7885 & 8531 \\
\hline New Zealand & EPNB & 565 & 544 & 542 & 522 & 552 & 584 & 592 & 600 & 667 & 745 \\
\hline South Korea & ERDY & 2784 & 3416 & 2756 & 2728 & 2563 & 3083 & 3063 & 3069 & 3073 & 3509 \\
\hline Oil exporting countries: & HCPC & 3228 & 4258 & 3969 & 3780 & 3923 & 4866 & 6017 & 6992 & 6387 & 8005 \\
\hline \multicolumn{12}{|l|}{ of which: } \\
\hline Brunei & QALG & 66 & 95 & 35 & 33 & 51 & 63 & 25 & 70 & 57 & 27 \\
\hline Dubai & QALU & 433 & 401 & 396 & 499 & 722 & 579 & 643 & 679 & 662 & 540 \\
\hline Indonesia & FKMS & 931 & 1081 & 1128 & 1006 & 875 & 918 & 839 & 958 & 925 & 1184 \\
\hline Kuwait & QATC & 121 & 314 & 296 & 271 & 313 & 396 & 367 & 741 & 696 & 1096 \\
\hline Nigeria & QATF & 112 & 89 & 65 & 90 & 83 & 106 & 152 & 206 & 271 & 912 \\
\hline Saudi Arabia & ERDU & 783 & 977 & 933 & 677 & 715 & 1158 & 1714 & 1232 & 821 & 673 \\
\hline Rest of the World & HCIF & 29973 & 36793 & 38352 & 38167 & 41800 & 47857 & 54388 & 60929 & 63727 & 75502 \\
\hline \multicolumn{12}{|l|}{ of which: } \\
\hline Brazil & FKMP & 910 & 1114 & 1279 & 1365 & 1477 & 1545 & 1740 & 1905 & 2061 & 2618 \\
\hline China & ERDZ & 3384 & 4826 & 5741 & 6726 & 8342 & 10390 & 12962 & 15237 & 18734 & 23159 \\
\hline Egypt & QALM & 255 & 411 & 406 & 416 & 432 & 495 & 349 & 662 & 538 & 638 \\
\hline Hong Kong & ERDS & 4909 & 5917 & 5754 & 5561 & 5500 & 5761 & 6602 & 7338 & 6939 & 8074 \\
\hline India & ERDV & 1426 & 1651 & 1816 & 1804 & 2093 & 2287 & 2781 & 3121 & 3809 & 4488 \\
\hline Israel & ERDX & 996 & 1025 & 939 & 880 & 861 & 920 & 1002 & 965 & 1045 & 1156 \\
\hline Malaysia & ERDW & 1961 & 2288 & 1939 & 1731 & 1867 & 2022 & 1813 & 1895 & 1684 & 1881 \\
\hline Pakistan & FKMV & 318 & 363 & 421 & 472 & 519 & 554 & 487 & 511 & 512 & 630 \\
\hline Philippines & FKMY & 983 & 1155 & 1155 & 944 & 713 & 655 & 712 & 742 & 717 & 629 \\
\hline Russia & EREC & 1324 & 1496 & 2047 & 1950 & 2454 & 3506 & 5010 & 5740 & 5248 & 6923 \\
\hline Singapore & ERDT & 2348 & 2395 & 2067 & 1959 & 2672 & 3379 & 3828 & 3756 & 4247 & 4008 \\
\hline South Africa & EPNE & 1636 & 2553 & 2841 & 2685 & 2949 & 3272 & 3937 & 3904 & 3060 & 4734 \\
\hline Taiwan & EREB & 2626 & 3561 & 2784 & 2385 & 2198 & 2341 & 2226 & 2339 & 2418 & 2597 \\
\hline Thailand & EREA & 1291 & 1602 & 1607 & 1550 & 1646 & 1760 & 1719 & 1922 & 2012 & 2427 \\
\hline
\end{tabular}

1 See chapter text.

2 Balance of payments consistent basis.

Source: Office for National Statistics: 02070142018

3 Includes Bulgaria and Romania after accession on 1 January 2007. 


\begin{tabular}{|c|c|c|c|}
\hline & Exports & Imports & Balances \\
\hline \multicolumn{4}{|l|}{ Agricultural,Mining and On-site Processing services } \\
\hline Agricultural & 24 & 72 & -48 \\
\hline Mining & 83 & 15 & 68 \\
\hline Waste treatment and depollution & 18 & 19 & -1 \\
\hline Other on-site processing services & 296 & 275 & 21 \\
\hline \multicolumn{4}{|l|}{ Business and Professional services } \\
\hline Advertising & 1922 & 1530 & 392 \\
\hline Management consulting & 1067 & 428 & 639 \\
\hline Public relations services & 150 & 41 & 108 \\
\hline Recruitment & 507 & 134 & 373 \\
\hline Other Business Management & 1245 & 803 & 442 \\
\hline Legal Services & 2589 & 535 & 2055 \\
\hline Market research and public opinion polling & 454 & 220 & 235 \\
\hline Operational leasing services & 349 & 425 & -76 \\
\hline Procurement & 102 & 150 & -48 \\
\hline Services between related enterprises & 5910 & 3426 & 2484 \\
\hline Other business and professional services & 1507 & 630 & 877 \\
\hline \multicolumn{4}{|l|}{ Communications services } \\
\hline Postal and courier & 358 & 534 & -177 \\
\hline Telecommunications & 3293 & 3044 & 249 \\
\hline \multicolumn{4}{|l|}{ Computer services } \\
\hline Computer & 4479 & 2260 & 2219 \\
\hline \multicolumn{4}{|l|}{ Information services } \\
\hline News agency services & 621 & 50 & 571 \\
\hline Publishing services & 294 & 84 & 210 \\
\hline Other information provision services & 1185 & 245 & 940 \\
\hline \multicolumn{4}{|l|}{ Construction Goods and Services } \\
\hline Construction in the UK & 188 & 326 & -138 \\
\hline Construction outside the UK & 603 & 339 & 264 \\
\hline \multicolumn{4}{|l|}{ Insurance Services } \\
\hline Auxiliary services & 1556 & 85 & 1471 \\
\hline Freight Insurance - Claims & 1 & & 1 \\
\hline Freigh Insurance - Premiums & & 7 & -7 \\
\hline Life insurance and pension funding - Claims & & & \\
\hline Life insurance and pension funding - Premiums & & 5 & -5 \\
\hline Reinsurance - Claims & 12 & & 12 \\
\hline Reinsurance - Premiums & & 23 & -23 \\
\hline Other Direct insurance - Claims & 26 & & 26 \\
\hline Other Direct insurance - Premiums & & 155 & -155 \\
\hline \multicolumn{4}{|l|}{ Merchanting and Other Trade related Services } \\
\hline Merchanting & .. & & .. \\
\hline Other trade related services & .. & 389 & .. \\
\hline \multicolumn{4}{|l|}{ Personal, Cultural and Recreational Services } \\
\hline Audio-Visual and related services & 198 & 66 & 132 \\
\hline Health services & 17 & 10 & 7 \\
\hline Training and educational services & 43 & 49 & -6 \\
\hline Other personal, cultural and recreational services & 338 & 159 & 180 \\
\hline \multicolumn{4}{|l|}{ Royalties and Licenses } \\
\hline Use of Franchise and similar rights fees & 1629 & 1896 & -267 \\
\hline Other royalties and license fees & 4589 & 1884 & 2705 \\
\hline Purchases and sales of franchises and similar right & 143 & 173 & -29 \\
\hline Purchases and sales of other royalties and licenses & 466 & 451 & 15 \\
\hline \multicolumn{4}{|l|}{ Technical services } \\
\hline Architectural & 177 & 22 & 156 \\
\hline Engineering & 3342 & 1279 & 2063 \\
\hline Surveying & 148 & 41 & 107 \\
\hline Other technical services & 1441 & 432 & 1009 \\
\hline \multicolumn{4}{|l|}{ Other Trade in Services } \\
\hline Other Trade in services & 2289 & 1163 & 1126 \\
\hline World Total & 59226 & 28379 & 30847 \\
\hline
\end{tabular}

1 Due to rounding, the sum of constituent items may not always equal the total shown.

2 Data excludes the following industries: Financial, Film and TV, Travel and Transport, Public Sector (including Education). Note (-) Denotes nil or less than $£ 500,000$. Note (..) Denotes disclosive data. 
European Union

Austria

Belgium

Cyprus

Czech Republic

Denmark

Estonia

Finland

France

Germany

Greece

Hungary

Irish Republic

Italy

Latvia

Lithuania

Luxembourg

Malta

Netherlands

Poland

Portugal

Slovakia

Slovenia

Spain

Sweden

EU Institutions

Total European Union

624

1156

69
149

556

23
616

2785

3724

217

204

3899

1484
22

22

650

21
4000

175

233

25
16

1258

719

22633

223

638

401

EFTA

Iceland

Liechtenstein

Norway

Switzerland

Total EFTA

63
33
823
3146
4065

$\begin{array}{r}73 \\ 85 \\ \hline\end{array}$

200

7
104

2662

3132

$\begin{array}{r}3126 \\ \hline\end{array}$

98

1184
996

8

178

13

1199

147

79
16

5
790

761

12732

Other European countries

Russia

Channel Islands

Isle of Man

Turkey

Rest of Europe

Europe Unallocated

Total Europe

\section{0}

440
734
88

144

358

1814

30278

$\begin{array}{rr}9 & 54 \\ 5 & 28 \\ 344 & 480 \\ 931 & 2215 \\ 1288 & 2777\end{array}$

Africa

Nigeria

South Africa

Rest of Africa

Africa Unallocated

298

2777

Total Africa

428
784
86

86
1597

128
264
16
157
125
935
15647

312

470

-13
-13

233

879

14631

America

Brazil

Canada

Mexico

USA

Rest of America

America Unallocated

Total America

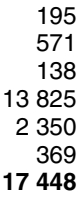

54
137
243
134
$\mathbf{5 6 8}$

245

291

541

1029 
Asia

China

Hong Kong

India

Indonesia

Israel

Japan

Malaysia

Pakistan

Phillippines

Saudi Arabia

Singapore

South Korea

Taiwan

Thailand

Rest of Asia

Asia Unallocated

134

281

353
463

463
77

166

1341

164

43
31

400

1851

303
170

78
1738

738

8761

342

418
39

39
156

915

38

17
25

530

340

47
46

29

243

Total Asia

4019

Australiasia and Oceania

Australia

New Zealand

Rest of Australia and Oceania

Oceania Unallocated

$\begin{array}{rrr}814 & 304 & 510 \\ 102 & 29 & 73 \\ 41 & 9 & 32 \\ 6 & 1 & 4 \\ 962 & 343 & 619\end{array}$

Total Australasia and Oceania

962

343

Rest of World Unallocated

International organisations

World Total

59226

28379

30847

Economic Zones

OECD

NAFTA

Central and Eastern Europe

42972

21831

21142

13764

$6478 \quad 7286$

OPEC

824

2745

2247

1030

NICs 1

2677

Offshore Financial centres

5376

ACP

1428

1 Due to rounding, the sum of constituent items may not always equal the to586

Source: Office for National Statistics: 01633456644 tal shown.

2 Data excludes the following industries: Financial, Film and TV, Travel and

Transport, Public Sector (including Education). Note (..) Denotes disclosive data. Note (-) Denotes nil or less than $£ 500,000$. 


\section{Current account}

A. Goods and services

1. Goods

2. Services

2.1. Transportation

15879

2.2. Travel

18313

2.3. Communications

3945

2.4. Construction

2.5. Insurance

4132

2.6. Financial

2.7. Computer and information

33832

2.8. Royalties and licence fees

6806

7471

37389

2.10. Personal, cultural and recreational

2136

2.11. Government

B. Income

238506

19032

34291

3683

1. Compensation of employees

2. Investment income

2.1 Direct investment

2.2 Portfolio investment

1058
237448

237448
84339

55122

97987

228409

2.3 Other investment (including earnings on reserve assets)

18223

1803

226606

52013

57490

117103

C. Current transfers

30147

1. General government

4351

13853

2. Other sectors

16294

Total current account

633113

678144

2. Capital and financial accounts

A. Capital account

1. Capital transfers

2. Acquisition/disposal of non-produced, non-financial assets

B. Financial account

1. Direct investment

Abroad

1.1. Equity capital

1.2. Reinvested earnings

1.3. Other capital ${ }^{2}$

In United Kingdom

1.1. Equity capital

1.2. Reinvested earnings

1.3. Other capital ${ }^{3}$

47155

25647

47795

$-26287$

2. Portfolio investment

Assets

2.1. Equity securities

2.2. Debt securities

Liabilities

2.1. Equity securities

2.2. Debt securities

3. Financial derivatives (net)

4. Other investment

Assets

56005

22930

1604

152609

138588

138588

19615

152609

$-11469$

164078

4.1 Trade credits

4.2 Loans

4.3 Currency and deposits

4.4 Other assets

Liabilities

4.1. Trade credits

4.2. Loans

4.3. Currency and deposits

4.4. Other liabilities

5. Reserve assets

5.1. Monetary gold

5.2. Special drawing rights

5.3. Reserve position in the IMF

5.4. Foreign exchange

Total capital and financial accounts

2 Other capital transaction on direct investment abroad represents claims on affiliated enterprises less liabilities to affiliated enterprises.

3 Other capital transactions on direct investment in the United Kingdom rep-

resents liabilities to direct investors less claims on direct investors. 


\begin{tabular}{|c|c|c|c|c|c|c|c|c|c|c|c|c|}
\hline & & & & & urrent account & & & & & & & \\
\hline & $\begin{array}{l}\text { Trade in } \\
\text { goods }\end{array}$ & $\begin{array}{l}\text { Trade in } \\
\text { services }\end{array}$ & $\begin{array}{r}\text { Total goods } \\
\text { and } \\
\text { services }\end{array}$ & $\begin{array}{r}\text { Compensati- } \\
\text { on of } \\
\text { employees }\end{array}$ & $\begin{array}{l}\text { Investment } \\
\text { income }\end{array}$ & $\begin{array}{r}\text { Total } \\
\text { income }\end{array}$ & $\begin{array}{l}\text { Current } \\
\text { transfers }\end{array}$ & $\begin{array}{l}\text { Current } \\
\text { balance }\end{array}$ & $\begin{array}{l}\text { Current } \\
\text { balance as } \\
\% \text { of GDP }{ }^{2}\end{array}$ & $\begin{array}{r}\text { Capital } \\
\text { account }\end{array}$ & $\begin{array}{r}\text { Financial } \\
\text { account }\end{array}$ & $\begin{array}{r}\text { Net errors } \\
\& \text { omissions }\end{array}$ \\
\hline & LQCT & KTMS & KTMY & KTMP & HMBM & HMBP & KTNF & HBOG & $\mathrm{AA} 6 \mathrm{H}$ & FKMJ & HBNT & $\mathrm{HHDH}$ \\
\hline 1954 & -210 & 115 & -95 & -27 & 227 & 200 & 55 & 160 & 0.9 & -13 & -174 & 27 \\
\hline 1955 & -315 & 42 & -273 & -27 & 149 & 122 & 43 & -108 & -0.6 & -15 & 34 & 89 \\
\hline 1956 & 50 & 26 & 76 & -30 & 203 & 173 & 2 & 251 & 1.2 & -13 & -250 & 12 \\
\hline 1957 & -29 & 121 & 92 & -32 & 223 & 191 & -5 & 278 & 1.3 & -13 & -313 & 48 \\
\hline 1958 & 34 & 119 & 153 & -34 & 261 & 227 & 4 & 384 & 1.7 & -10 & -411 & 37 \\
\hline 1959 & -116 & 118 & 2 & -37 & 233 & 196 & - & 198 & 0.8 & -5 & -68 & -125 \\
\hline 1960 & -404 & 39 & -365 & -35 & 201 & 166 & -6 & -205 & -0.8 & -6 & -7 & 218 \\
\hline 1961 & -144 & 51 & -93 & -35 & 223 & 188 & -9 & 86 & 0.3 & -12 & 23 & -97 \\
\hline 1962 & -104 & 50 & -54 & -37 & 301 & 264 & -14 & 196 & 0.7 & -12 & -195 & 11 \\
\hline 1963 & -123 & 4 & -119 & -38 & 364 & 326 & -37 & 170 & 0.6 & -16 & -30 & -124 \\
\hline 1964 & -551 & -34 & -585 & -33 & 365 & 332 & -74 & -327 & -1.0 & -17 & 392 & -48 \\
\hline 1967 & -601 & 157 & -444 & -39 & 354 & 315 & -118 & -247 & -0.6 & -25 & 179 & 93 \\
\hline 1968 & -708 & 341 & -367 & -48 & 303 & 255 & -119 & -231 & -0.5 & -26 & 688 & -431 \\
\hline 1969 & -214 & 392 & 178 & -47 & 468 & 421 & -109 & 490 & 1.0 & -23 & -794 & 327 \\
\hline 1970 & -18 & 457 & 437 & -56 & 527 & 471 & -89 & 819 & 1.6 & -22 & -818 & 21 \\
\hline 1971 & 205 & 617 & 822 & -63 & 454 & 391 & -90 & 1123 & 2.0 & -23 & -1330 & 230 \\
\hline 1972 & -736 & 722 & -14 & -52 & 350 & 298 & -142 & 142 & 0.2 & -35 & 477 & -584 \\
\hline 1973 & -2573 & 907 & -1666 & -68 & 970 & 902 & -336 & -1100 & -1.5 & -39 & 1031 & 108 \\
\hline 1974 & -5241 & 1292 & -3949 & -92 & 1010 & 918 & -302 & -3333 & -4.0 & -34 & 3185 & 182 \\
\hline 1975 & -3245 & 1708 & -1537 & -102 & 257 & 155 & -313 & -1695 & -1.6 & -36 & 1569 & 162 \\
\hline 1976 & -3930 & 2872 & -1058 & -140 & 760 & 620 & -534 & -972 & -0.8 & -12 & 507 & 477 \\
\hline 1977 & -2271 & 3704 & 1433 & -152 & -678 & -830 & -889 & -286 & -0.2 & 11 & -3286 & 3561 \\
\hline 1978 & -1534 & 4215 & 2681 & -140 & -300 & -440 & -1420 & 821 & 0.5 & -79 & -2655 & 1913 \\
\hline 1979 & -3326 & 4573 & 1247 & -130 & -342 & -472 & -1777 & -1002 & -0.5 & -103 & 864 & 241 \\
\hline 1980 & 1329 & 4414 & 5743 & -82 & -2268 & -2350 & -1653 & 1740 & 0.8 & -4 & -2157 & 421 \\
\hline 1981 & 3238 & 4776 & 8014 & -66 & -1883 & -1949 & -1219 & 4846 & 1.9 & -79 & -5312 & 545 \\
\hline 1982 & 1879 & 4261 & 6140 & -95 & -2336 & -2431 & -1476 & 2233 & 0.8 & 6 & -1233 & -1006 \\
\hline 1984 & -5409 & 6101 & 692 & -94 & -326 & -420 & -1566 & -1294 & -0.4 & 107 & -7130 & 8317 \\
\hline 1985 & -3416 & 8499 & 5083 & -120 & -2609 & -2729 & -2924 & -570 & -0.2 & 185 & -1657 & 2042 \\
\hline 1986 & -9617 & 8182 & -1435 & -156 & 71 & -85 & -2094 & -3614 & -0.9 & 135 & -122 & 3601 \\
\hline 1987 & -11698 & 8604 & -3094 & -174 & -730 & -904 & -3437 & -7435 & -1.7 & 333 & 10606 & -3504 \\
\hline 1988 & -21553 & 6388 & -15165 & -64 & -1188 & -1252 & -3293 & -19710 & -4.1 & 235 & 16989 & 2486 \\
\hline 1989 & -24724 & 5866 & -18858 & -138 & -2309 & -2447 & -4228 & -25533 & -4.9 & 270 & 13614 & 11649 \\
\hline 1990 & -18707 & 6643 & -12064 & -110 & -4586 & -4696 & -4802 & -21562 & -3.8 & 497 & 22272 & -1207 \\
\hline 1991 & -10223 & 6312 & -3911 & -63 & -5642 & -5705 & -999 & -10615 & -1.8 & 290 & 7855 & 2470 \\
\hline 1992 & -13050 & 6353 & -6697 & -49 & -1037 & -1086 & -5228 & -13011 & -2.1 & 421 & 16311 & -3721 \\
\hline 1993 & -13066 & 8174 & -4892 & 35 & -2547 & -2512 & -5056 & -12460 & -1.9 & 309 & 22278 & -10127 \\
\hline 1994 & -11126 & 8161 & -2965 & -170 & 1521 & 1351 & -5187 & -6801 & -1.0 & 33 & -3240 & 10008 \\
\hline 1995 & -12023 & 11165 & -858 & -296 & -546 & -842 & -7363 & -9063 & -1.2 & 533 & -1717 & 10247 \\
\hline 1996 & -13722 & 14312 & 590 & 93 & -2460 & -2367 & -4539 & -6316 & -0.8 & 1260 & -940 & 5996 \\
\hline 1997 & -12342 & 16801 & 4459 & 83 & 241 & 324 & -5745 & -962 & -0.1 & 958 & -7294 & 7298 \\
\hline 1998 & -21813 & 15003 & -6810 & -10 & 11813 & 11803 & -8172 & -3179 & -0.4 & 489 & 4480 & -1790 \\
\hline 1999 & -29051 & 15562 & -13489 & 201 & -1244 & -1043 & -7322 & -21854 & -2.4 & 747 & 29505 & -8398 \\
\hline 2000 & -32976 & 15002 & -17974 & 150 & 1812 & 1962 & -9775 & -25787 & -2.6 & 1703 & 23133 & 951 \\
\hline 2001 & -41212 & 17200 & -24012 & 66 & 9359 & 9425 & -6515 & -21102 & -2.1 & 1318 & 27194 & -7410 \\
\hline 2002 & -47705 & 19632 & -28073 & 67 & 18219 & 18286 & -8870 & -18657 & -1.7 & 932 & 24204 & -6479 \\
\hline 2003 & -48607 & 22612 & -25995 & 59 & 17464 & 17523 & -9835 & -18307 & -1.6 & 1466 & 22553 & -5712 \\
\hline 2004 & -60900 & 28146 & -32754 & -494 & 18324 & 17830 & -10276 & -25200 & -2.1 & 2064 & 19564 & 3572 \\
\hline 2005 & -68589 & 25875 & -42714 & -610 & 22482 & 21872 & -11849 & -32691 & -2.6 & 1503 & 30581 & 607 \\
\hline 2006 & -76312 & 33108 & -43204 & -745 & 10842 & 10097 & -11924 & -45031 & -3.4 & 975 & 41875 & 2181 \\
\hline 2007 & -89754 & 42457 & -47297 & -665 & 21237 & 20572 & -13602 & -40327 & -2.9 & 2566 & 39712 & -1951 \\
\hline 2008 & -92876 & 48878 & -43998 & -619 & 33748 & 33129 & -13624 & -24493 & -1.7 & 3505 & 19657 & 1331 \\
\hline
\end{tabular}

1 See chapter text.

2 Using series YBHA: GDP at current market prices. 


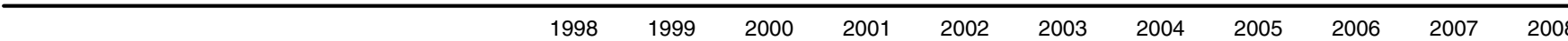

Credits

Exports of goods and services

Exports of goods

Exports of

Total exports of goods and services KTMW

Income

Compensation of employees

Investment income

Total income

KTMN

HMBN

HMBQ

Current transfers

General government

Other sectors

Total current transfers

Total

Debits

Imports of goods and services Imports of goods

Imports of services

LQBI

KTMR

Total imports of goods and services KTMX

Income

Compensation of employees

Investment income

Total income

KTMO

HMBO

HMBR

Current transfers

General government

Other sectors

Total current transfers

Total

HBOF

Balances

Trade in goods and services

Trade in goods

Trade in services

Total trade in goods and services

LOCT

KTMS

KTMY

Income

Compensation of employees

Investment income

Total income

KTMP

HMBM

HMBP

Current transfers

General government

Other sectors

Total current transfers

Total (Current balance)

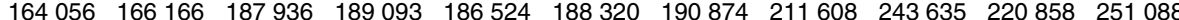
\begin{tabular}{lllllllllllllll}
69228 & 76525 & 81883 & 87773 & 94 & 012 & 102357 & 112518 & 119420 & 132749 & 149191 & 165944 \\
\hline
\end{tabular} $\overline{233284} \overline{242691} \overline{269819} \overline{276866} \overline{280536} \overline{290677} \overline{303392} \overline{331028} \overline{376384} \overline{370049} \overline{417032}$

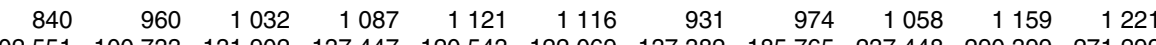
$\begin{array}{llllllllllll}102551 & 100733 & 131902 & 137447 & 120543 & 122069 & 137382 & 185765 & 237448 & 290399 & 271 & 299\end{array}$

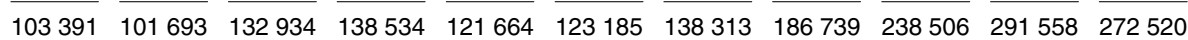

\begin{tabular}{|c|c|c|c|c|c|c|c|c|c|c|}
\hline $\begin{array}{r}1767 \\
10682\end{array}$ & $\begin{array}{l}3542 \\
8510\end{array}$ & $\begin{array}{l}2465 \\
8018\end{array}$ & $\begin{array}{l}4991 \\
8926\end{array}$ & $\begin{array}{l}3663 \\
8571\end{array}$ & $\begin{array}{l}3968 \\
8079\end{array}$ & $\begin{array}{l}4177 \\
9590\end{array}$ & $\begin{array}{r}4294 \\
13106\end{array}$ & $\begin{array}{r}4351 \\
13872\end{array}$ & $\begin{array}{l}4346 \\
9731\end{array}$ & $\begin{array}{r}5625 \\
10126\end{array}$ \\
\hline 12449 & 12052 & 10483 & 13917 & 12234 & 12047 & 13767 & 17400 & 18223 & 14077 & 157 \\
\hline 349124 & $\overline{356436}$ & $\overline{413236}$ & $\overline{429317}$ & $\overline{414434}$ & $\overline{425909}$ & $\overline{455472}$ & 535167 & $\overline{633113}$ & $\overline{675684}$ & \\
\hline
\end{tabular}

$\begin{array}{llllllllllll}185869 & 195217 & 220912 & 230305 & 234229 & 236927 & 251774 & 280197 & 319947 & 310612 & 343964\end{array}$ $\begin{array}{lllllllllll}54225 & 60963 & 66881 & 70573 & 74380 & 79745 & 84372 & 93545 & 99641 & 106734 & 117066\end{array}$

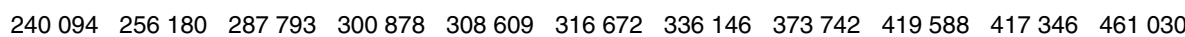

$\begin{array}{lllllllllll}850 & 759 & 882 & 1021 & 1054 & 1057 & 1425 & 1584 & 1803 & 1824 & 1840\end{array}$ $90738 \quad 101977 \quad 130090 \quad 128088 \quad 102324 \quad 104605 \quad 119058 \quad 163283226606 \quad 269162 \quad 237551$

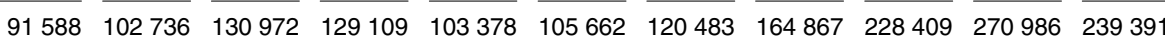

\begin{tabular}{|c|c|c|c|c|c|c|c|c|c|}
\hline $\begin{array}{r}6585 \\
14036\end{array}$ & $\begin{array}{r}7271 \\
12103\end{array}$ & $\begin{array}{r}7778 \\
12480\end{array}$ & $\begin{array}{r}7340 \\
13092\end{array}$ & $\begin{array}{r}9085 \\
12019\end{array}$ & $\begin{array}{l}10657 \\
11225\end{array}$ & $\begin{array}{l}12225 \\
11818\end{array}$ & $\begin{array}{l}13637 \\
15612\end{array}$ & $\begin{array}{l}13853 \\
16294\end{array}$ & $\begin{array}{l}14082 \\
13597\end{array}$ \\
\hline 20621 & 19374 & 20258 & 20432 & 21104 & 21882 & 24043 & 29249 & 30147 & 27679 \\
\hline 52303 & 378290 & 439023 & 450419 & 433091 & 444216 & 480672 & 567858 & 678144 & 716011 \\
\hline
\end{tabular}

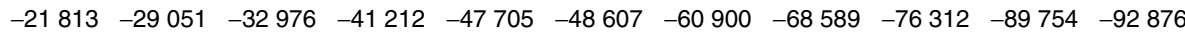
$\begin{array}{lllllllllll}15003 & 15562 & 15002 & 17200 & 19632 & 22612 & 28146 & 25875 & 33108 & 42457 & 48878\end{array}$ $\begin{array}{lllllllllll}-6810 & \overline{-13489} & \overline{-17974} & \overline{-24012} & \overline{-28073} & \overline{-25995} & -32754 & -42714 & -43204 & \overline{-47297} & \overline{-43998}\end{array}$

\begin{tabular}{|c|c|c|c|c|c|c|c|c|c|c|}
\hline $\begin{array}{r}-10 \\
813\end{array}$ & $\begin{array}{r}201 \\
-1244\end{array}$ & $\begin{array}{r}150 \\
1812\end{array}$ & $\begin{array}{r}66 \\
9359\end{array}$ & $\begin{array}{r}67 \\
18219\end{array}$ & $\begin{array}{r}59 \\
17464\end{array}$ & $\begin{array}{r}-494 \\
18324\end{array}$ & $\begin{array}{r}-610 \\
22-482\end{array}$ & $\begin{array}{r}-745 \\
10842\end{array}$ & $\begin{array}{r}-665 \\
21237\end{array}$ & $\begin{array}{r}-619 \\
33748\end{array}$ \\
\hline 11803 & -1043 & 1962 & 9425 & 18286 & 17523 & 17830 & 21872 & 10097 & 20572 & 3129 \\
\hline
\end{tabular}

$\begin{array}{lllllllllllll}\text { FJUQ } & -4818 & -3729 & -5313 & -2349 & -5422 & -6689 & -8048 & -9343 & -9502 & -9736 & -8993\end{array}$ $\begin{array}{llllllllllll}\text { FJUR } & -3354 & -3593 & -4462 & -4166 & -3448 & -3146 & -2228 & -2506 & -2422 & -3866 & -4631\end{array}$ $\begin{array}{lllllllllllllllll}\text { KTNF } & -8172 & -7322 & -9775 & \overline{-6515} & -8870 & -9835 & \overline{-10276} & \overline{-11849} & \overline{-11924} & -13602 & \overline{-13624}\end{array}$

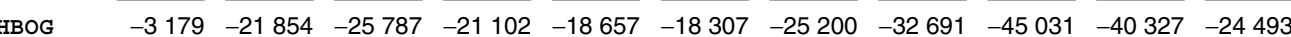


2000

2001

2002

2003

2004

2005

2006

\section{Investment abroad}

International investment position Direct investment

Portfolio investment

Other investment

Reserve assets

Total

Financial account transactions

Direct investment

Portfolio investment

Financial derivatives (net)

Other investment

Reserve assets

Total

Investment income

Direct investment

Portfolio investment

Other investment

Reserve assets

Total

Investment in the UK

International investment position

Direct investment

Portfolio investment

Other investment

Total

Financial account transactions

Direct investment

Portfolio investment

Other investment

Total

Investment income

Direct investment

Portfolio investment

Other investment

Total

Net investment

International investment position Direct investment

Portfolio investment

Other investment

Reserve assets

Net investment position

Financial account transactions

Direct investment

Portfolio investment

Financial derivatives

Other investment

Reserve assets

Net transactions

nvestment income

Direct investment

Portfolio investment

Other investment

Reserve assets

Net earnings

1 See chapter text.
HBWI

HLXW

HLYD

HBOB

HJYU

HHZF

XBMN

HBNS

HJYX

HLZC

HLZN

HMBO

HBWQ

CGNH

CGNG

LTEB

HBQC

HJYV

HHZD

ZPNN

HHYR

HBNT

HJYE

HLZX

CGNA

HHCB

HMBM

\begin{tabular}{rrrrrrrrrrr}
309.8 & 438.3 & 618.8 & 616.9 & 637.2 & 691.1 & 678.1 & 705.9 & 733.6 & 913.9 & 1036.2 \\
703.8 & 838.3 & 906.1 & 937.4 & 844.0 & 935.8 & 1092.3 & 1361.2 & 1531.1 & 1686.2 & 1751.3 \\
1098.4 & 1097.3 & 1379.7 & 1521.9 & 1545.2 & 1813.7 & 2118.1 & 2714.7 & 2917.3 & 3750.7 & 4316.7 \\
23.3 & 22.2 & 28.8 & 25.6 & 25.5 & 23.8 & 23.2 & 24.7 & 22.9 & 26.7 & 36.3 \\
\hline 2135.4 & 2396.1 & 2933.4 & 3101.9 & 3051.9 & 3464.5 & 3911.7 & 4806.6 & 5204.9 & 6377.5 & 7140.5
\end{tabular}

\begin{tabular}{|c|c|c|c|c|c|c|c|c|c|c|}
\hline 73.8 & 125.6 & 155.6 & 42.8 & 35.0 & 40.9 & 51.5 & 44.0 & 47.2 & 136.1 & 60.3 \\
\hline 32.1 & 21.4 & 65.6 & 86.6 & 1.0 & 36.3 & 140.9 & 151.0 & 138.6 & 92.0 & -132.0 \\
\hline 3.0 & -2.7 & -1.6 & -8.4 & -1.0 & 5.4 & 7.9 & -9.6 & -7.4 & 19.0 & -18.1 \\
\hline 30.0 & 41.5 & 241.7 & 170.7 & 70.4 & 260.4 & 325.3 & 501.2 & 395.7 & 747.1 & -347.1 \\
\hline-0.2 & -0.6 & 3.9 & -3.1 & -0.5 & -1.6 & 0.2 & 0.7 & -0.4 & 1.2 & -1.3 \\
\hline 138.7 & 185.2 & 465.2 & 288.5 & 105.0 & 341.4 & 525.8 & 687.4 & 573.6 & 995.4 & -438.3 \\
\hline
\end{tabular}

\begin{tabular}{|c|c|c|c|c|c|c|c|c|c|c|}
\hline 29.9 & 33.1 & 45.0 & 46.7 & 51.5 & 55.1 & 63.3 & 79.2 & 84.3 & 90.3 & 77.7 \\
\hline 29.3 & 25.9 & 33.0 & 34.9 & 32.5 & 32.5 & 36.7 & 45.4 & 55.1 & 66.1 & 67.9 \\
\hline 42.2 & 40.6 & 52.9 & 54.9 & 35.8 & 33.6 & 36.7 & 60.5 & 97.3 & 133.4 & 124.9 \\
\hline 1.1 & 1.2 & 1.0 & 1.0 & 0.8 & 0.8 & 0.7 & 0.7 & 0.6 & 0.6 & 0.8 \\
\hline 102.6 & 10 & 131.9 & 137.4 & 120.5 & 122. & 137.4 & 185.8 & 237.4 & 290.4 & 71.3 \\
\hline
\end{tabular}

$\begin{array}{rrrrrrrrrrr}213.6 & 250.2 & 310.4 & 363.5 & 340.6 & 355.5 & 383.3 & 494.2 & 577.4 & 630.2 & 674.2 \\ 739.9 & 933.2 & 1067.6 & 1013.2 & 925.3 & 1082.9 & 1227.6 & 1457.5 & 1704.3 & 1918.2 & 1981.4\end{array}$

\begin{tabular}{llllllllllll}
1350.3 & 1400.9 & 1651.6 & 1861.9 & 1906.0 & 2143.2 & 2520.8 & 3103.0 & 3293.8 & 4126.4 & 4551.3 \\
\hline
\end{tabular}

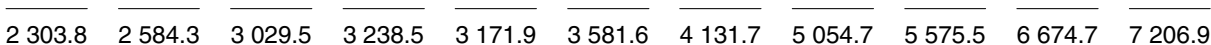

\begin{tabular}{|c|c|c|c|c|c|c|c|c|c|c|}
\hline 45.1 & 55.1 & 80.6 & 37.3 & 16.8 & 16.8 & 31.2 & 97.8 & 80.5 & 98.2 & 52.4 \\
\hline 25.0 & 106.3 & 172.2 & 40.8 & 49.7 & 105.6 & 88.5 & 132.7 & 152.6 & 208.7 & 257.5 \\
\hline 73.1 & 53.3 & 235.6 & 237.6 & 62.6 & 241.5 & 425.6 & 487.4 & 382.3 & 728.3 & -728.5 \\
\hline & 214.7 & 488.3 & 315.7 & 129.2 & 364.0 & 545.3 & 718.0 & 615.5 & 035.1 & - \\
\hline
\end{tabular}

\begin{tabular}{|c|c|c|c|c|c|c|c|c|c|c|}
\hline 8.6 & 17.0 & 27.4 & 21.4 & 16.0 & 21.9 & 27.6 & 36.2 & 52.0 & 44.8 & 11.9 \\
\hline 29.5 & 32.2 & 32.4 & 36.1 & 33.3 & 32.9 & 38.7 & 47.6 & 57.5 & 66.5 & 74.2 \\
\hline 52.7 & 52.7 & 70.2 & 70.5 & 53.0 & 49.8 & 52.7 & 79.6 & 117.1 & 157.8 & 151.4 \\
\hline 0.7 & 102.0 & 130.1 & 128.1 & 102.3 & 104.6 & 119.1 & 163.3 & 226.6 & 269.2 & 237.6 \\
\hline
\end{tabular}

\begin{tabular}{rrrrrrrrrrr}
96.2 & 188.1 & 308.4 & 253.5 & 296.6 & 335.6 & 294.7 & 211.7 & 156.2 & 283.8 & 362.0 \\
-36.0 & -94.9 & -161.5 & -75.7 & -81.3 & -147.0 & -135.2 & -96.3 & -173.2 & -232.0 & -230.1 \\
-251.9 & -303.6 & -271.9 & -339.9 & -360.8 & -329.5 & -402.7 & -388.3 & -376.5 & -375.7 & -234.6 \\
23.3 & 22.2 & 28.8 & 25.6 & 25.5 & 23.8 & 23.2 & 24.7 & 22.9 & 26.7 & 36.3 \\
\hline-168.4 & -188.2 & -96.2 & -136.5 & -120.0 & -117.2 & -220.0 & -248.2 & -370.7 & -297.2 & -66.4
\end{tabular}

\begin{tabular}{|c|c|c|c|c|c|c|c|c|c|c|}
\hline-28.7 & -70.5 & -75.0 & -5.5 & -18.3 & -24.1 & -20.3 & 53.8 & 33.4 & -38.0 & -7.9 \\
\hline-7.0 & 84.9 & 106.6 & -45.7 & 48.7 & 69.4 & -52.3 & -18.3 & 14.0 & 116.7 & 389.5 \\
\hline-3.0 & 2.7 & 1.6 & 8.4 & 1.0 & -5.4 & -7.9 & 9.6 & 7.4 & -19.0 & 18.1 \\
\hline 43.1 & 11.8 & -6.1 & 66.9 & -7.7 & -18.9 & 100.2 & -13.8 & -13.4 & -18.8 & -381.5 \\
\hline 0.2 & 0.6 & -3.9 & 3.1 & 0.5 & 1.6 & -0.2 & -0.7 & 0.4 & -1.2 & 1.3 \\
\hline 4.5 & 29.5 & 23.1 & 27.2 & 24.2 & 22.6 & 19.6 & 30.6 & 41.9 & 39.7 & 19 \\
\hline
\end{tabular}

$\begin{array}{rrrrrrrrrrr}21.3 & 16.1 & 17.6 & 25.3 & 35.5 & 33.2 & 35.7 & 43.0 & 32.3 & 45.4 & 65.8 \\ -0.2 & -6.4 & 0.5 & -1.2 & -0.8 & -0.4 & -2.0 & -2.2 & -2.4 & -0.4 & -6.3 \\ -10.5 & -12.2 & -17.3 & -15.7 & -17.2 & -16.1 & -16.0 & -19.0 & -19.8 & -24.4 & -26.5 \\ 1.1 & 1.2 & 1.0 & 1.0 & 0.8 & 0.8 & 0.7 & 0.7 & 0.6 & 0.6 & 0.8 \\ & -1.2 & 1.8 & 9.4 & 18.2 & 17.5 & 18.3 & 22.5 & 10.8 & 21.2 & 33.7\end{array}$


$£$ million

\begin{tabular}{|c|c|c|c|c|c|c|}
\hline & & 2003 & 2004 & 2005 & 2006 & 2007 \\
\hline Europe & GQBX & 16600 & 10814 & 12105 & 16899 & 60983 \\
\hline EU27 & IY6N & 13417 & 11917 & 13337 & 4038 & 53042 \\
\hline Austria & CBJD & 165 & 1322 & -301 & -94 & 148 \\
\hline Belgium & HIIL & -1241 & -544 & 970 & -4356 & 1127 \\
\hline Bulgaria & IY 60 & .. & - & 11 & -5 & \\
\hline Cyprus & DG8D & -53 & 18 & 69 & 98 & 365 \\
\hline Czech Republic & DG80 & 142 & 23 & 24 & -160 & 128 \\
\hline Denmark & CAUW & -53 & 569 & 391 & 1529 & -69 \\
\hline Finland & CBJE & 99 & -37 & $70 \overline{7}$ & 106 & 275 \\
\hline France & CAUX & 6627 & 793 & 3138 & 1175 & 3427 \\
\hline Germany & CAUY & 1552 & -366 & -479 & 3186 & 1634 \\
\hline Greece & CAUZ & 229 & -253 & 63 & 15 & 105 \\
\hline Hungary & DG8F & 527 & 336 & 1821 & 39 & 144 \\
\hline Irish Republic & CAVA & 985 & 3325 & -1181 & 5161 & 4269 \\
\hline Italy & CAVB & 500 & 667 & 191 & -397 & 1752 \\
\hline Latvia & DG8G & .. & 1 & -1 & 4 & .. \\
\hline Lithuania & DG8H & .. & 1 & -4 & 1 & - \\
\hline Luxembourg & HIIM & 1313 & -1022 & -1213 & -14131 & 8879 \\
\hline Netherlands & CAVC & 728 & 4805 & 4821 & 1350 & 25347 \\
\hline Poland & DG8J & 4 & 182 & 150 & 397 & -568 \\
\hline Portugal & CAVD & 308 & 444 & 603 & 314 & 529 \\
\hline Romania & IY6P & 56 & 11 & 101 & 40 & 120 \\
\hline Slovakia & DG8K & -11 & 18 & 21 & 18 & 87 \\
\hline Slovenia & DG8L & 37 & -5 & -5 & 14 & 9 \\
\hline Spain & CAVE & 626 & 1131 & 564 & 2177 & 4143 \\
\hline Sweden & CBJG & 794 & 299 & 2732 & 6669 & 3262 \\
\hline $\begin{array}{l}\text { EFTA } \\
\text { of which }\end{array}$ & CAVG & 2313 & -6667 & 547 & 6926 & 3309 \\
\hline Norway & CBJF & -274 & 367 & -831 & 3 & 1057 \\
\hline Switzerland & CBJH & 2591 & -7007 & 1330 & 6948 & 2345 \\
\hline $\begin{array}{l}\text { Other European Countries } \\
\text { of which }\end{array}$ & IY6Q & 870 & 5564 & -1779 & 5935 & 4631 \\
\hline Russia & GLAA & 2030 & 1831 & 349 & -13 & 1340 \\
\hline UK offshore islands & GLAC & -1031 & 3528 & -2341 & 5023 & 2088 \\
\hline Brazil & CBLA & 786 & 386 & 48 & 354 & 721 \\
\hline Canada & CAVK & 2521 & 1143 & 3372 & 8130 & 21865 \\
\hline Chile & GQCA & 290 & 675 & 790 & 25 & 106 \\
\hline Colombia & $\mathrm{GQCB}$ & 78 & 225 & -687 & 315 & 110 \\
\hline Mexico & GLAD & 261 & 1386 & 168 & 334 & 96 \\
\hline Panama & GLAE & 58 & 12 & 27 & 7 & -18 \\
\hline USA & CAVJ & 19300 & 9732 & 15041 & -1803 & 32255 \\
\hline Asia & GQCI & 3601 & 7689 & 5399 & 7992 & 9214 \\
\hline $\begin{array}{l}\text { Near and Middle East Countries } \\
\text { of which }\end{array}$ & CBKF & 82 & 486 & 398 & 1219 & 1912 \\
\hline Gulf Arabian countries ${ }^{3}$ & GQCC & -85 & 293 & 577 & 329 & 343 \\
\hline $\begin{array}{l}\text { Other Asian Countries } \\
\text { of which }\end{array}$ & GQCD & 3518 & 7203 & 5001 & 6773 & 7302 \\
\hline China & HIIN & 309 & 539 & 598 & 374 & 1142 \\
\hline Hong Kong & CAVN & 1285 & 5303 & 1547 & 1674 & 1744 \\
\hline India & GLAF & 193 & 274 & 616 & 104 & 667 \\
\hline Indonesia & GLAG & 481 & -289 & -116 & 196 & -134 \\
\hline Japan & CAVM & 338 & 37 & 247 & 440 & 2278 \\
\hline Malaysia & CBKN & 277 & 428 & 244 & 241 & 263 \\
\hline Singapore & CBKQ & -449 & -161 & -508 & 2621 & -1215 \\
\hline South Korea & GLAH & 332 & 278 & 2247 & 679 & 488 \\
\hline Thailand & GLAI & 155 & 181 & 228 & 536 & 5 \\
\hline $\begin{array}{l}\text { Australasia and Oceania } \\
\text { of which }\end{array}$ & GQCE & -1524 & 1026 & 423 & 3132 & 1187 \\
\hline Australia & CBJO & -492 & 408 & 444 & 2743 & 1289 \\
\hline New Zealand & CBJP & -1017 & 258 & -56 & 405 & -116 \\
\hline $\begin{array}{l}\text { Africa } \\
\quad \text { of which }\end{array}$ & GQCF & 3454 & 5863 & 5843 & -235 & 4728 \\
\hline Kenya & GLAJ & 58 & 47 & 73 & 62 & 96 \\
\hline Nigeria & CBJY & 19 & -44 & -108 & 44 & 671 \\
\hline South Africa & CAVO & 2222 & 3840 & 4368 & 1466 & 1105 \\
\hline Zimbabwe & CBKD & 37 & 91 & 18 & 8 & 5 \\
\hline World Total & CDQD & 38088 & 49713 & 44458 & 46887 & 137678 \\
\hline
\end{tabular}

1 See chapter text. Net investment includes re-invested earnings.

4 From 2003 includes Albania, Bosnia \& Herzegovina, Bulgaria, Croatia, FYR of

2 Minus sign indicates net disinvestment abroad.

3 Includes Abu Dhabi, Bahrain, Dubai, Iraq, Kuwait, Oman, Other Gulf States, Macedonia, Romiania and Serbia \& Montenegro. Prior to 2003 Czech Republic, Estonia, Hungary, Latvia, Lithuania, Poland, Slovakia and Slovenia also included. 

book value of net assets: by area and main country ${ }^{1}$

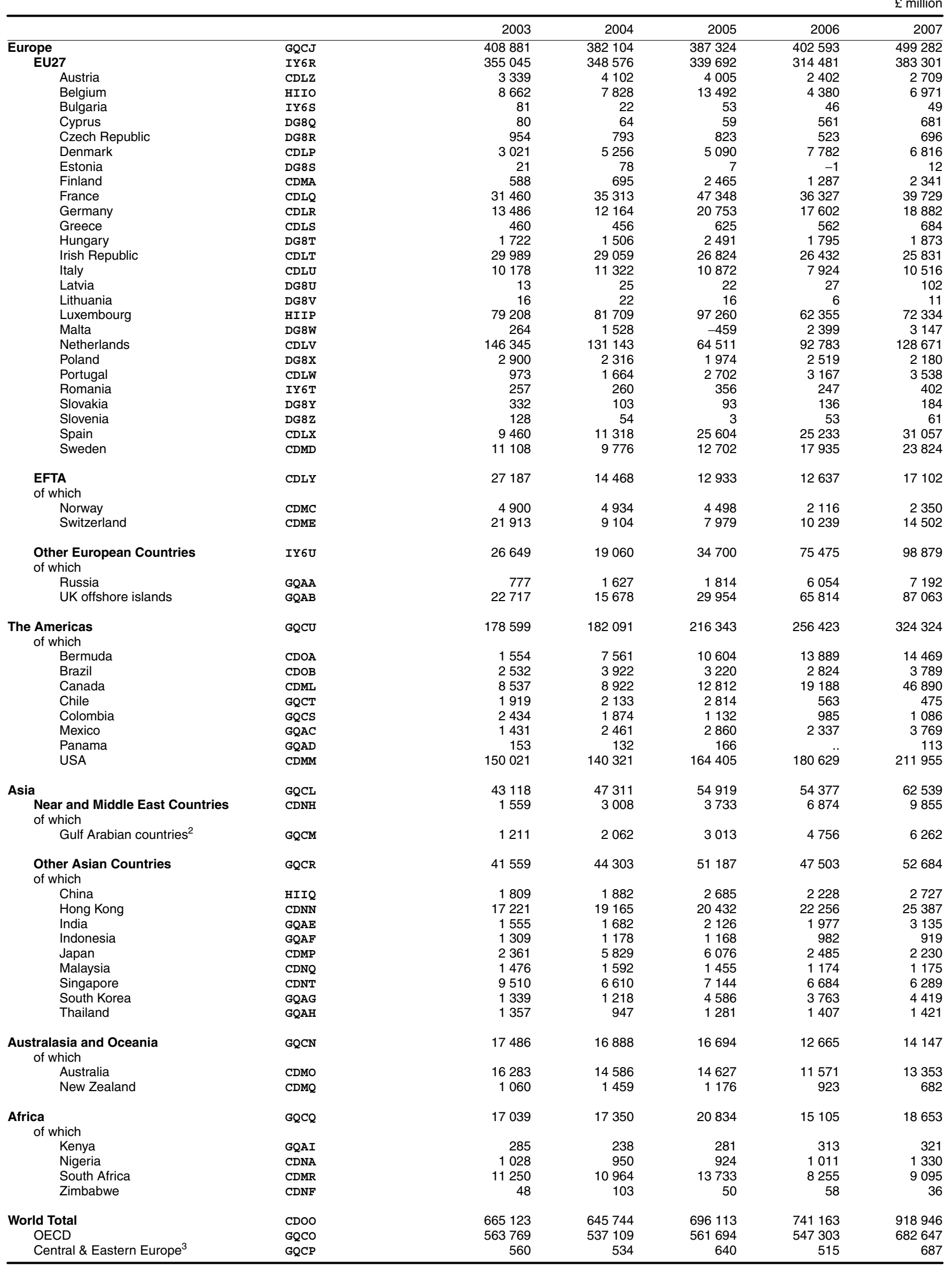

1 See chapter text.

2 Includes Abu Dhabi, Bahrain, Dubai, Iraq, Kuwait, Oman, Other Gulf States,

Republic, Estonia

Qatar, Saudi Arabia and Yemen.

also included.

3 From 2003 includes Albania, Bosnia \& Herzegovina, Bulgaria, Croatia, FYR

of Macedonia, Romiania and Serbia \& Montenegro. Prior to 2003 Czech 


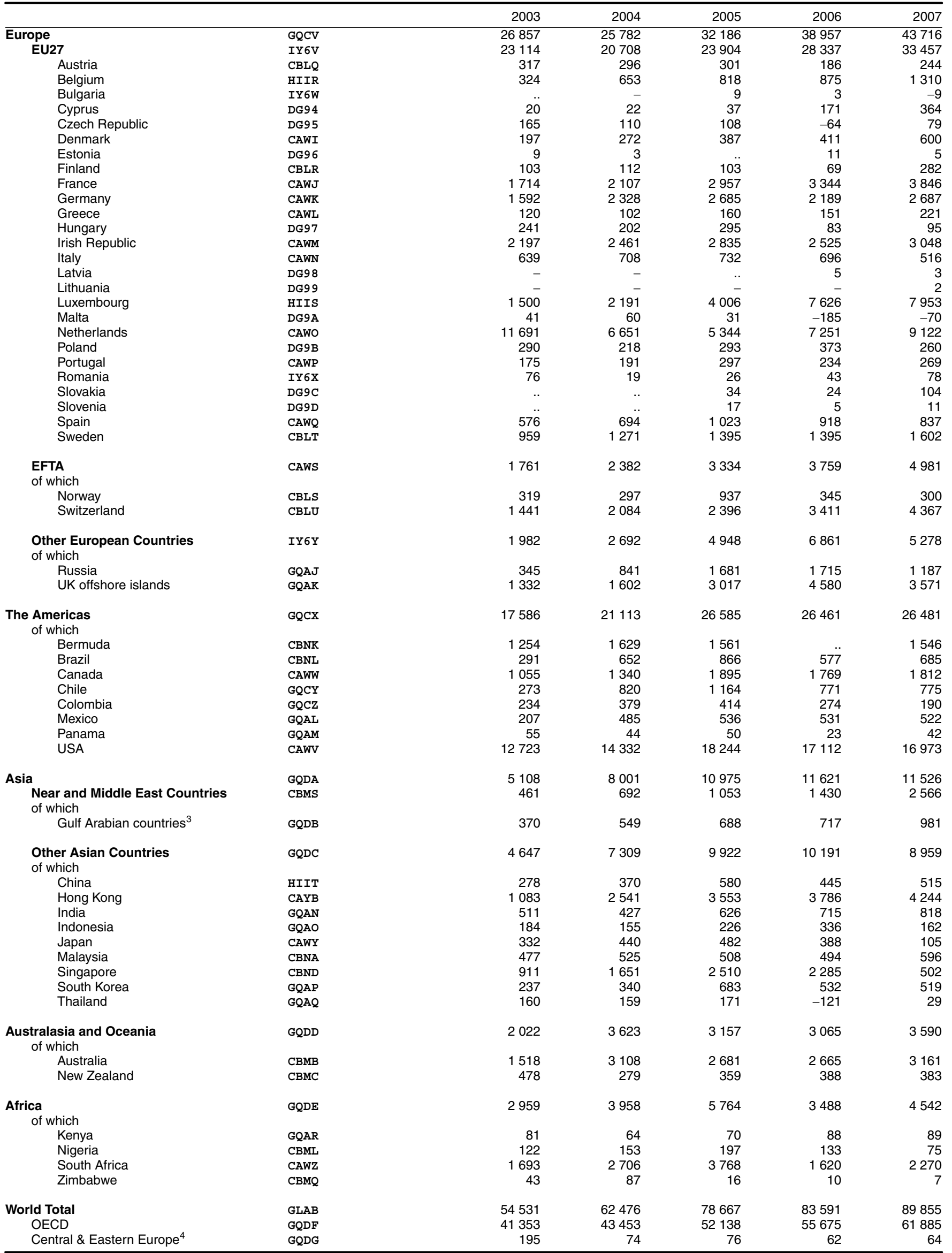

1 See chapter text. A minus sign indicates net losses.

3 Includes Abu Dhabi, Bahrain, Dubai, Iraq, Kuwait, Oman, Other Gulf States,

2 Net earnings equal profits of overseas branches plus UK companies' reQatar, Saudi Arabia and Yemen.

ceipts of interest and their share of profits of overseas subsidiaries and as- 4 From 2003 includes Albania, Bosnia \& Herzegovina, Bulgaria, Croatia, FYR of sociates. Earnings are after deducting provisions for depreciation and with- Macedonia, Romiania and Serbia \& Montenegro. Prior to 2003 Czech Repubholding tax on profits, dividends and interest. 
External trade and investment

\begin{tabular}{|c|c|c|c|c|c|c|}
\hline & & 2003 & 2004 & 2005 & 2006 & 2007 \\
\hline Europe & GQDH & 7013 & 29901 & 80087 & 53837 & 48696 \\
\hline EU27 & IY6Z & 5505 & 26384 & 71034 & 47698 & 37818 \\
\hline Austria & СВОВ & 8 & -31 & 171 & -61 & 153 \\
\hline Belgium & HIIU & 218 & 1542 & 23 & 670 & \\
\hline Bulgaria & IY72 & .. & .. & & & \\
\hline Cyprus & DG9G & - & - & 7 & 18 & 75. \\
\hline Czech Republic & DG9H & - & - & - & & 1 \\
\hline Denmark & CAYQ & 321 & -11 & -1246 & 13 & \\
\hline Estonia & DG9I I & - & - & - & - & \\
\hline Finland & CBOC & 26 & 32 & 238 & 44 & \\
\hline France & CAYR & 414 & 1703 & 9643 & 2356 & -2057 \\
\hline Germany & CAYS & 1437 & 11131 & 7279 & 5566 & 1882 \\
\hline Greece & CAYT & 33 & 13 & 14 & 17 & 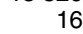 \\
\hline Hungary & DG9J & - & & 1 & 3 & \\
\hline Irish Republic & CAYU & 206 & 936 & 723 & 816 & 185 \\
\hline Italy & CAYV & -468 & 1327 & -42 & 282 & $2 \varepsilon$ \\
\hline Latvia & DG9K & .. & - & .. & .. & \\
\hline Lithuania & DG9L & - & .. & - & - & \\
\hline Luxembourg & HIIV & -105 & -115 & 151 & 221 & 33 \\
\hline Malta & DG9M & - & - & 1 & 2 & \\
\hline Netherlands & CAYW & 2452 & 1226 & 50366 & 13715 & 15 \\
\hline Poland & DG9N & - & - & 1 & 50 & -2 \\
\hline Portugal & CAYX & .. & & -6 & 9 & 1 \\
\hline Romania & IY73 & .. & -34 & .. & .. & \\
\hline Slovakia & DG9O & .. & .. & .. & .. & \\
\hline Slovenia & DG9P & & .. & & & \\
\hline Spain & CAYY & 518 & & 3297 & 23457 & 148 \\
\hline Sweden & СВOE & 406 & -14 & 393 & 508 & \\
\hline $\begin{array}{l}\text { EFTA } \\
\text { of which }\end{array}$ & CAZB & 1408 & 3016 & 9050 & 5321 & 93 \\
\hline Norway & CBOD & -179 & -798 & 927 & 171 & \\
\hline Switzerland & CBOF & 1411 & 3488 & 7405 & 4786 & 86 \\
\hline $\begin{array}{l}\text { Other European Countries } \\
\text { of which }\end{array}$ & IY74 & 100 & 501 & 3 & 817 & \\
\hline Russia & GQAS & & & & & \\
\hline UK offshore islands & GQAT & 32 & 476 & -60 & 733 & 14 \\
\hline $\begin{array}{c}\text { The Americas } \\
\text { of which }\end{array}$ & GQDJ & 3396 & -4792 & 17422 & 17242 & $30 \mathrm{~s}-\mathrm{s} \cdot \mathrm{s} \cdot \mathrm{s} \cdot \mathrm{s}$ \\
\hline Brazil & HP5A & 4 & & 6 & & \\
\hline Canada & CAZF & -325 & 683 & 1632 & 3509 & \\
\hline USA & CAZE & 2676 & -5727 & 15589 & 12313 & 271 \\
\hline Asia & GQDK & -449 & 4081 & -4168 & 11806 & 11 \\
\hline Near and Middle East Countries & GQAU & -34 & 384 & 736 & 5034 & \\
\hline $\begin{array}{l}\text { Other Asian Countries } \\
\text { of which }\end{array}$ & GQAV & -415 & 3697 & -4904 & 6772 & 106 \\
\hline China & HР5B & 2 & -26 & 13 & 12 & \\
\hline Hong Kong & GQAW & 63 & & 315 & 92 & \\
\hline India & $\mathrm{HP} 5 \mathrm{C}$ & 7 & -15 & 138 & 265 & \\
\hline Japan & CAZH & -543 & 817 & -5575 & 3726 & 58 \\
\hline Singapore & GQAX & -76 & 14 & 46 & & \\
\hline South Korea & GQAY & -20 & 193 & 175 & -85 & \\
\hline $\begin{array}{l}\text { Australasia and Oceania } \\
\text { of which }\end{array}$ & GQDL & 310 & 1420 & 3396 & 1869 & \\
\hline Australia & Свол & 309 & 1412 & 3396 & 1479 & \\
\hline New Zealand & СBOK & 2 & 8 & - & 54 & \\
\hline $\begin{array}{l}\text { Africa } \\
\text { of which }\end{array}$ & GQAZ & 7 & -43 & 66 & 131 & \\
\hline South Africa & CAZJ & 21 & -35 & 25 & 101 & \\
\hline World Total & $\mathrm{CBDH}$ & 10276 & 30566 & 96803 & 84885 & 91651 \\
\hline OECD & GQBA & 8984 & 26762 & 95187 & 73961 & 811 \\
\hline Central \& Eastern Europe ${ }^{3}$ & $\mathrm{GQBB}$ & 4 & -32 & & 6 & \\
\hline
\end{tabular}

1 See chapter text. Net investment includes reinvested earnings.

2 A minus sign indicates net disinvestment in the UK.

Sources: ONS Foreign Direct Investment Surveys: 01633 456647;

3 From 2003 includes Albania, Bosnia \& Herzegovina, Bulgaria, Croatia, FYR

of Macedonia, Romiania and Serbia \& Montenegro. Prior to 2003 Czech

Republic, Estonia, Hungary, Latvia, Lithuania, Poland, Slovakia and

Slovenia also included. 
1047 United Kingdom inward foreign direct international investment position:

. book value of net liabilities: by area and main country

At year end

$£$ million

\begin{tabular}{|c|c|c|c|c|c|c|}
\hline & & 2003 & 2004 & 2005 & 2006 & 2007 \\
\hline Europe & GQDM & 158903 & 181198 & 277027 & 332077 & 361925 \\
\hline EU27 & IY75 & 142273 & 161395 & 244392 & 299906 & 314695 \\
\hline Austria & CDPF & 349 & 366 & 561 & 848 & 969 \\
\hline Belgium & HIIW & 1987 & 4338 & 4481 & 5609 & 3925 \\
\hline Bulgaria & IY76 & & & & & \\
\hline Cyprus & DG9S & 67 & 78 & 100 & 162 & 437 \\
\hline Czech Republic & DG9T & 9 & 6 & 3 & & 8 \\
\hline Denmark & CDOV & 2086 & 2359 & 1404 & 4344 & 5353 \\
\hline Estonia & DG9U & - & - & - & - & \\
\hline Finland & CDPG & 946 & 886 & 756 & 817 & 759 \\
\hline France & CDOW & 36565 & 41100 & 56309 & 59998 & 54120 \\
\hline Germany & CDOX & 32260 & 39300 & 51469 & 54382 & 70284 \\
\hline Greece & CDOY & 86 & 100 & 103 & 121 & 165 \\
\hline Hungary & DG9V & 9 & 12 & 9 & 12 & 12 \\
\hline Irish Republic & $\mathrm{CDOZ}$ & 4769 & 5021 & 7146 & 8186 & 7971 \\
\hline Italy & CDPA & 4580 & 6708 & 6122 & 4482 & 4640 \\
\hline Latvia & DG9W & .. & - & .. & .. & .. \\
\hline Lithuania & DG9X & & & - & - & 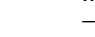 \\
\hline Luxembourg & HIIX & 5627 & 5963 & 7880 & 16021 & 18537 \\
\hline Malta & DG9Y & 21 & 5 & 12 & 12 & 62 \\
\hline Netherlands & CDPB & 46876 & 47579 & 95579 & 119843 & 113462 \\
\hline Poland & DG9Z & 10 & 7 & 21 & 96 & 74 \\
\hline Portugal & CDPC & 115 & 113 & 111 & 122 & 222 \\
\hline Romania & IY77 & .. & .. & .. & .. & .. \\
\hline Slovakia & DGA2 &.. &.. & - & $\ddot{-}$ & .. \\
\hline Slovenia & DGA3 & & & & & 9 \\
\hline Spain & CDPD & 3344 & 4536 & 8782 & 20658 & 29290 \\
\hline Sweden & CDPI & 2527 & 2849 & 3467 & 4113 & 4302 \\
\hline $\begin{array}{l}\text { EFTA } \\
\text { of which }\end{array}$ & CDPE & 13758 & 15752 & 25033 & 22358 & 34606 \\
\hline Norway & $\mathrm{CDPH}$ & 831 & 242 & 1085 & 969 & 1348 \\
\hline Switzerland & CDPJ & 12439 & 14685 & 21624 & 19033 & 31149 \\
\hline $\begin{array}{l}\text { Other European Countries } \\
\text { of which }\end{array}$ & IY78 & 2872 & 4051 & 7602 & 9813 & 12624 \\
\hline Russia & GQBC & & & & & 179 \\
\hline UK offshore islands & GQBD & 2361 & 3500 & 7059 & 9111 & 11741 \\
\hline $\begin{array}{c}\text { The Americas } \\
\text { of which }\end{array}$ & GQDU & 145973 & 140090 & 174037 & 200709 & 201871 \\
\hline Brazil & HP 5D & 5 & & 77 & 134 & 21 \\
\hline Canada & CDPM & 11176 & 12108 & 15587 & 19369 & 20528 \\
\hline USA & CDPN & 130512 & 122069 & 149759 & 170880 & 167610 \\
\hline Asia & GQDO & 19869 & 24800 & 24101 & 39436 & 56499 \\
\hline Near and Middle East Countries & GQBE & 1627 & 2765 & 2970 & 10160 & 10631 \\
\hline $\begin{array}{l}\text { Other Asian Countries } \\
\text { of which }\end{array}$ & GQBF & 18242 & 22035 & 21131 & 29275 & 45868 \\
\hline China & HP5E & 102 & 119 & 111 & 99 & 193 \\
\hline Hong Kong & GQBG & & & & & \\
\hline India & HP5F & 194 & 164 & 518 & 798 & 1221 \\
\hline Japan & CDPQ & 11949 & 12300 & 10513 & 14766 & 25206 \\
\hline Singapore & GQBH & 830 & 925 & 1034 & 4046 & \\
\hline South Korea & GQBI & 635 & 635 & 638 & 798 & 793 \\
\hline $\begin{array}{l}\text { Australasia and Oceania } \\
\text { of which }\end{array}$ & GQDP & 14336 & 16804 & 12537 & 7623 & 9096 \\
\hline Australia & CDPP & 14160 & 16631 & 12313 & 7093 & 8655 \\
\hline New Zealand & CDPR & 158 & 153 & 224 & 428 & 433 \\
\hline $\begin{array}{l}\text { Africa } \\
\text { of which }\end{array}$ & GQBJ & 560 & 530 & 510 & 469 & 1362 \\
\hline South Africa & CDPS & 387 & 296 & 186 & 130 & 868 \\
\hline World Total & CDPZ & 339641 & 363422 & 488212 & 580313 & 630753 \\
\hline OECD & GQBK & 324491 & 340870 & 458185 & 535218 & 571652 \\
\hline Central \& Eastern Europe ${ }^{2}$ & GQBL & 34 & .. & .. & .. & 93 \\
\hline
\end{tabular}

1 See chapter text.

2 From 2003 includes Albania, Bosnia \& Herzegovina, Bulgaria, Croatia, FYR Sources: ONS Foreign Direct Investment Surveys 01633 456647; of Macedonia, Romiania and Serbia \& Montenegro. Prior to 2003 Czech Republic, Estonia, Hungary, Latvia, Lithuania, Poland, Slovakia and Slovenia also included. 
External trade and investment $1918 \begin{aligned} & \text { Net earnings from foreign direct investment in the United Kingdom: }:^{1,2} \\ & \text { by area and main country }\end{aligned}$

\begin{tabular}{|c|c|c|c|c|c|c|}
\hline & & 2003 & 2004 & 2005 & 2006 & 2007 \\
\hline Europe & GQDQ & 10158 & 12676 & 17592 & 27447 & 25254 \\
\hline EU27 & IYั79 & 9010 & 11330 & 15278 & 22919 & 22913 \\
\hline Austria & CBOR & 111 & 61 & 60 & 207 & 212 \\
\hline Belgium & HIIY & 165 & 269 & 367 & 646 & 408 \\
\hline Bulgaria & IY7A & - &.. & - & - & \\
\hline Cyprus & DGA6 & 24 & 20 & 24 & 44 & 66 \\
\hline Czech Republic & DGA7 & - & - & - &.. & 1 \\
\hline Denmark & CBDL & 305 & 311 & 326 & 204 & 298 \\
\hline Estonia & DGA8 & - & - & - & - & \\
\hline Finland & CBOS & 36 & 62 & 61 & 93 & 181 \\
\hline France & CBDM & 2743 & 3842 & 5121 & 5329 & 3063 \\
\hline Germany & CBDN & 1754 & 2900 & 4037 & 4541 & 5791 \\
\hline Greece & CBDO & 4 & -4 & 49 & 70 & 104 \\
\hline Hungary & DGA 9 & - & - & 1 & 3 & 1 \\
\hline Irish Republic & CBDP & 578 & 471 & 724 & 1012 & 119 \\
\hline Italy & CBDQ & 174 & 408 & 483 & 477 & 57 \\
\hline Latvia & DGB2 &.. & - &.. &.. & \\
\hline Lithuania & DGB3 & - & .. & 1 & 1 & \\
\hline Malta & DGB4 & - & - & - & 3 & $f$ \\
\hline Netherlands & CBDR & 2405 & 2585 & 2800 & 7283 & 8331 \\
\hline Poland & DGB5 & - & - & 1 & 8 & 6 \\
\hline Portugal & CBDS & 49 & 47 & 30 & 48 & 54 \\
\hline Romania & IY7B & .. &.. & .. & .. & \\
\hline Slovakia & DGB 6 &.. & .. & 5 & 5 & 3 \\
\hline Slovenia & DGB7 &.. & .. &.. & .. & \\
\hline Spain & CBDT & 298 & 37 & 773 & 2536 & 1610 \\
\hline Sweden & CBOU & 167 & 21 & 182 & 316 & 59 \\
\hline $\begin{array}{l}\text { EFTA } \\
\text { of which }\end{array}$ & CBDW & 822 & 849 & 1495 & 3366 & \\
\hline Norway & СВОт & - & -20 & 82 & 169 & 19 \\
\hline Switzerland & CBOV & 794 & 819 & 1320 & 2933 & -27 \\
\hline $\begin{array}{l}\text { Other European Countries } \\
\text { of which }\end{array}$ & IY7C & 326 & 497 & 819 & 1162 & 2065 \\
\hline Russia & GQBM &.. &.. & .. & .. & 7 \\
\hline UK offshore islands & GQBN & 287 & 468 & 757 & 1107 & 1999 \\
\hline Brazil & HP 5G &.. & 1 & -4 & -4 & 6 \\
\hline Canada & CBEA & 639 & 1021 & 1348 & 1458 & -252 \\
\hline USA & CBDZ & 9054 & 10981 & 14156 & 16828 & 1535 \\
\hline Asia & GQDS & -781 & 168 & 937 & 2710 & 701 \\
\hline Near and Middle East Countries & GQBO & 114 & 188 & 354 & 564 & 49 \\
\hline $\begin{array}{l}\text { Other Asian Countries } \\
\text { of which }\end{array}$ & GQBP & -895 & -19 & 583 & 2145 & 2 \\
\hline China & HP 5H & & 8 & -63 & -35 & 17 \\
\hline Hong Kong & GQBQ & -456 & .. & .. & -597 & \\
\hline India & HP5I & 55 & -2 & 65 & 132 & 139 \\
\hline Japan & CBEC & -538 & 608 & 1089 & 1956 & -244 \\
\hline Singapore & GQBS & 62 & 32 & 85 & 259 & 588 \\
\hline South Korea & GQिBT & -54 & 23 & 72 & 104 & 125 \\
\hline $\begin{array}{l}\text { Australasia and Oceania } \\
\text { of which }\end{array}$ & GQDT & 835 & 695 & 535 & 1259 & 1227 \\
\hline Australia & $\mathrm{CBOZ}$ & 802 & 690 & 521 & 876 & 1010 \\
\hline New Zealand & CBPA & 4 & 5 & 13 & 46 & 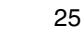 \\
\hline $\begin{array}{l}\text { Africa } \\
\text { of which }\end{array}$ & GQBU & 59 & 59 & 65 & 80 & 1 \\
\hline South Africa & CBED & 50 & 26 & 25 & 31 & 97 \\
\hline World Total & CBEV & 20283 & 25876 & 35588 & 51650 & 44821 \\
\hline OECD & GQBV & 19715 & 25471 & 33927 & 47476 & 39097 \\
\hline Central \& Eastern Europe 3 & GQBW & 1 & 1 & .. &.. & 6 \\
\hline
\end{tabular}

1 See chapter text. A minus sign indicates net losses.

2 Net earnings equal profits of UK branches plus overseas investors' receipts

of interest and their share of the profits of UK subsidiaries and associates.

Sources: ONS Foreign Direct Investment Surveys: 01633 456647; Earnings are after deducting provisions for depreciation and withholding tax on profits and interest.

3 From 2003 includes Albania, Bosnia \& Herzegovina, Bulgaria, Croatia, FYR of Macedonia, Romiania and Serbia \& Montenegro. Prior to 2003 Czech Republic, Estonia, Hungary, Latvia, Lithuania, Poland, Slovakia and

Slovenia also included. 